\title{
Estimating hemodynamic risks of tandem stenoses in posterior cerebral circulation: a computational study
}

Haipeng Liu

Coventry University

Defeng Wang

Beihang University

Xinyi Leng

Chinese University of Hong Kong

Linfang Lan

Chinese University of Hong Kong

Vicent Hing Lung Ip

Chinese University of Hong Kong

\section{Lawrence Ka Sing Wong}

Chinese University of Hong Kong

Dingchang Zheng

Coventry University

Fei Chen

Southern University of Science and Technology

Lin Shi ( $\nabla$ shilin@cuhk.edu.hk)

https://orcid.org/0000-0003-2318-4669

Thomas Wai Hong Leung

Chinese University of Hong Kong

\section{Research}

Keywords: posterior cerebral circulation, tandem stenoses, fractional flow (FF), computational fluid dynamics (CFD), low-density lipoprotein (LDL)

Posted Date: March 18th, 2020

DOI: https://doi.org/10.21203/rs.3.rs-17625/v1

License: (a) (i) This work is licensed under a Creative Commons Attribution 4.0 International License.

Read Full License 



\section{RESEARCH}

\section{Estimating hemodynamic risks of tandem stenoses in posterior cerebral circulation: a computational study}

Haipeng Liu ${ }^{1,2,3}$, Defeng Wang ${ }^{2,4,5,6}$, Xinyi Leng ${ }^{3}$, Linfang Lan ${ }^{3}$, Vincent Hing Lung Ip ${ }^{3}$, Lawrence Ka Sing Wong ${ }^{3}$, Dingchang Zheng ${ }^{1}$, Fei Chen ${ }^{7}$, Lin Shi ${ }^{2 * \dagger}$ and Thomas Wai Hong Leung ${ }^{3 \dagger}$

\footnotetext{
${ }^{*}$ Correspondence: shilin@cuhk.edu.hk

${ }^{2}$ Department of Imaging and Interventional Radiology, The Chinese University of Hong Kong, Prince of Wales Hospital, Hong Kong, China

Full list of author information is available at the end of the article

$\dagger$ Corresponding Authors
}

\begin{abstract}
Background: Tandem stenoses were widely observed in acute stroke cases. High pressure drop and low fractional flow (FF) indicate the risk of ischemia caused by intracranial atherosclerotic stenosis. Low wall shear stress (WSS) and high low-density lipoprotein (LDL) filtration rate indicate the risk of atherosclerotic plaque growth. High WSS is related to the risk of plaque rupture. The hemodynamic parameters (pressure drop, FF, WSS, and LDL filtration rate) could be estimated using computational fluid dynamics (CFD) simulation. Few studies have investigated the hemodynamics of intracranial tandem stenoses. This study aims to estimate the clinical risks of intracranial tandem stenoses using CFD simulation.
\end{abstract}

Methods: With identical radius and length, 4 cylindrical tube models were developed with different stenoses (severity in area: $75 \%$ single, $75 \%-84 \%$ tandem, $75 \%-84 \%-89 \%$ tandem, and $89 \%$ single). The pressure drop and FF of each stenosis were derived from static CFD simulation and compared between models. A transient CFD simulation was performed on a patient-specific intracranial arterial model with three $(56.2 \%, 51.3 \%$, and $89.8 \%)$ tandem stenoses using boundary conditions derived from patient-specific measurements. For the three stenoses, pressure drop, FF, WSS, and LDL filtration rate were calculated and compared.

Results: Independent from other stenoses, $89 \%$ single stenosis caused comparable pressure drops in four tube models (difference less than 10\%), larger than those of $75 \%-84 \%$ tandem stenoses. $89 \%$ stenosis had the lowest FF (less than 0.85$)$. In the patient-specific model, the $56.2 \%$ and $51.3 \%$ stenoses had pressure drops less than $2 \mathrm{mmHg}$ with FFs higher than 0.98 , while the $89.8 \%$ stenosis caused a pressure drop more than $10 \mathrm{mmHg}$ with FF lower than 0.90 , where a high WSS belt extended distally. Compared with low WSS (less than $0.5 \mathrm{~Pa}$ ), high LDL filtration rate appeared in smaller areas on arterial wall, with less temporal fluctuations.

Conclusions: 1. Tandem stenoses are independent in pressure drop and FF. Pressure drop increases sharply with the severity of stenosis. 2. Compared with low WSS, high LDL filtration rate is more specific and stable in reflecting the risk of intracranial atherosclerotic plaque growth. 3 . The risks of both plaque growth and rupture should be considered in severe stenoses.

Keywords: posterior cerebral circulation; tandem stenoses; fractional flow (FF); computational fluid dynamics (CFD); low-density lipoprotein (LDL) 


\section{Background}

Intracranial atherosclerotic stenosis (ICAS) is a common cause of stroke. It can cause transient ischemic attack, with a high morbidity and serious sequelae such as dementia[1]. Especially, a high rate of tandem stenoses was observed in acute stroke cases with ICAS[2]. Basilar artery (BA) is the main artery of posterior cerebral circulation, and the second most common site of ICAS[3]. However, few studies have investigated the effect of tandem intracranial stenoses on the clinical risks (ischemia, growth and rupture of atherosclerotic plaques) of ICAS, especially in BA.

It has been known that hemodynamic parameters are associated with clinical risks of ICAS. For an intracranial stenosis, a high trans-stenosis pressure drop and low fractional flow (FF) indicate the risk of ischemia[4]. Low wall shear stress (WSS) has been proven to be related with the growth of atherosclerotic plaques while high WSS indicates the risk of plaque rapture[5]. High filtration rate of low-density lipoprotein (LDL) through endothelial promotes, therefore reflects the risk of, atherosclerotic plaque growth[6].

These hemodynamic parameters (pressure drop, FF, WSS, and LDL filtration rate) are difficult to measure in intracranial arteries, but could be simulated in arterial models using computational fluid dynamics (CFD). Pilot studies have validated the application of CFD simulation in intracranial arteries [7], especially in posterior cerebral circulation[8]. The pressure and FF values in BA derived by CFD simulation accorded with patient-specific measurements[9]. However, there is a lack of study that comprehensively estimates the effect of tandem stenoses on different hemodynamic parameters and clinical risks in posterior cerebral circulation.

In this study, we focused on the effect of tandem stenoses on these hemodynamic parameters, to estimate the related risks of ischemia, plaque growth, and plaque rupture in posterior cerebral circulation.

\section{Results}

Pressure drop and FF in tube models

The area-averaged pressure was calculated on the cross-sections every $10 \mathrm{~mm}$ from the inlet. Fig. 1 illustrates the diastolic pressure distribution. The extended line of curve 1 indicates the reference pressure drop along the tube without stenoses. Due to hemodynamic effects, the pressure dropped at the stenotic throat and increased to a static value in the distal segment, which is most obvious in the $89 \%$ stenosis. Therefore the measurement sites $(10 \mathrm{~mm}$ proximal and $20 \mathrm{~mm}$ distal to the stenotic throat) to calculate pressure drop are reasonable.

It can be observed that pressure drops of tandem stenoses were independent and accumulative. In Fig. 1, 75\%-84\% tandem stensoses cause $6 \mathrm{mmHg}$ pressure drop in model 3. The difference in pressure drop between model 2 (with $89 \%$ stenosis) and model 4 (with $75 \%-84 \%-89 \%$ tandem stenoses) is about $6.5 \mathrm{mmHg}$, with a relative difference less than $10 \%$ compared with the $6 \mathrm{mmHg}$ pressure drop in model 3 . Similarly, the pressure drop caused by the single $89 \%$ stenosis (distance between line 1 and its extension) is nearly equal (relative difference $<10 \%$ ) to the difference in pressure drop between models 3 (with 75\%-85\% tandem stenoses) and model 4 (with 75\%-84\%-89\% tandem stenoses). 
Moreover, the pressure drop of a stenosis depends non-linearly on the severity. The pressure drop caused by $89 \%$ stenosis (distance between line 1 and its extension) is more than that caused by $75 \%-84 \%$ tandem stenoses (distance between line 3 and the extension of line 1). The pressure drop of $75 \%$ stenosis (distance between line 2 and the extension of line 1 ) is less than $2 \mathrm{mmHg}$. We inferred that a stenosis with less than $75 \%$ lumen occlusion has negligible pressure drop, with $\mathrm{FF}>0.9$.

Table 1 lists the diastolic and systolic FF values. The FF values for the $75 \%$ stenosis are nearly identical in models 2,3 , and 4 , in diastole and systole respectively. Similarly, the $84 \%$ stenosis has comparable FF values in models 3 and 4 , so does the $89 \%$ stensosis in models 1 and 4 . Therefore, in tandem stenoses, the FF value of a certain stenosis is independent from other stenoses.

\section{Pressure drop and FF in patient-specific model}

In both end-of-diastolic (Fig. 2a) and systolic (Fig. 2b) pressure distributions, the blood pressure decreased smoothly along the arteries except at the third stenosis (severity in area: $89.8 \%$, cross-section 12 in Fig. 2c and d). As in tube models, the pressure decreased at the stenotic throat but increased in distal segment. In Table 2, the $56.2 \%$ and $51.3 \%$ stenoses have pressure drops less than $2 \mathrm{mmHg}$ with FF values higher than 0.98 . Comparatively, the $89.8 \%$ stenosis has a much higher pressure drop ( $>8 \mathrm{mmHg}$ in diastole, $>15 \mathrm{mmHg}$ in systole), with $\mathrm{FF}<0.87$. This accorded with the conclusion drawn from the tube models that a stenosis less than $75 \%$ in area has negligible pressure drop with $\mathrm{FF}>0.9$.

\section{WSS distribution in patient-specific model}

In Fig. 3, WSS shows obvious difference between diastole and systole in most areas. However, some areas have constantly high or low WSS values. High WSS $(>4.5 \mathrm{~Pa})$ exists at three stenotic throats while low WSS $(<0.5 \mathrm{~Pa})$ exists in the distal segments of stenoses 1 and 3. In the extended WSS distribution (Fig. 4), the red belts indicated the distribution of high WSS at three stenotic throats due to the jet flow with high velocity. Distal to the first stenosis there is an independent speck of high WSS area, reflecting the impact of diverted upstream jet flow, due to the bend proximal to the first stenosis. With the least severity ( $51.3 \%$ in area), the second stenosis has the least obvious high-WSS belt. The third stenosis has the highest severity $(89.8 \%$ in area), with an obvious high-WSS belt. At the third stenosis, the jet flow is diverted due to the bend. Consequently, the high-WSS belt expands distally along one side of the arterial wall.

From the data in Table 3, the ratio between diastolic and systolic mean WSS values was calculated in different areas. This WSS ratio was comparable in different areas (difference within $\pm 7.5 \%$ ), indicating the global influence of temporal flow fluctuations on WSS. Compared with the whole arterial wall, stenotic areas 1 and 2 showed similar WSS statistics while stenotic area 3 had much higher mean WSS ( $>4$ folds of mean WSS in any other area) with higher SD.

\section{LDL filtration rate distribution in patient-specific model}

Spatially, compared with WSS (Fig. 3), the LDL filtration rate (Fig. 5) shows the less obvious difference between diastolic and systolic distributions. The areas 
with high LDL filtration rate often had low WSS values. However, low WSS values $(<0.5 \mathrm{~Pa})$ existed in many segments, while high $\mathrm{LDL}$ values $(>4.5 \mathrm{~Pa})$ concentrated in several areas, especially the arterial segment distal to the first stenosis. LDL filtration rate indicated more concentrated risky areas of plaque growth.

Temporally, we compared the transient fluctuations of WSS and LDL filtration of a point on the arterial segment distal to the first stenosis where high LDL filtration rate and low WSS exist (Fig. 6). WSS and LDL filtration rate showed opposite fluctuation trends, with the waveform different from the inlet pressure (Fig. 8). The fluctuations of WSS and LDL filtration rate are not proportional. From the normalized values, it is obvious the temporal fluctuations of LDL filtration rate were much smoother (maximum/average ratio $<2$ ) than those of WSS (maximum/average ratio $>5.5)$.

\section{Discussion}

In this pilot study, we investigated the effect of tandem stenoses on the clinical risks in posterior cerebral circulation, including ischemia (indicated by high pressure drop and low FF), atherosclerotic plaque growth (indicated by low WSS and high LDL filtration rate), and plaque rupture (indicated by high WSS). The results of CFD simulation showed independent hemodynamic effects of tandem stenoses on the clinical risks.

As to the risk of ischemia, the tube and patient-specific models derived the same conclusion. In tube models, the effects of tandem stenoses on the pressure and FF were independent and accumulative. The pressure drop increased sharply with severity of stenosis. The most severe stenosis (89\%) caused a larger pressure drop than the tandem $75 \%-84 \%$ stenoses (Fig. 1). A theoretical study showed that a tandem of $70 \%-80 \%$ stenoses had similar effect to that of a single $82 \%$ stenosis[10]. We suggested that the stenoses with $75 \%$ or lower severity in area causes limited risk of ischemia, which is confirmed by the patient-specific model where only the most severe stenosis $(89.8 \%)$ had an obvious pressure drop with $\mathrm{FF}<0.9$. These results were in accordance with the clinical diagnostic criterion which focuses exclusively on stenosis with $>70 \%$ severity in diameter[4].

In the patient-specific model, the difference between diastolic and systolic FFs was within $\pm 4 \%$ in all the three stenoses. Parallel studies in coronary arteries had similar conclusions[11]. Therefore, $\mathrm{FF}$ is a hemodynamic parameter that reliably reflects the risk of ischemia, without being affected by flow rate. We suggest that static simulation is able to substitute transient simulation in estimating the FF of a stenosis less than $85 \%$ in area.

As to the risk of atherosclerotic plaque growth, in the patient-specific model, high LDL filtration rate shows more specific areas in spatial distribution (Fig. 5 and Fig. 3) with less temporal fluctuations (Fig. 6) compared with low WSS $(<0.5 \mathrm{~Pa})$. Low WSS promotes atherosclerosis by changing the permeability of endothelium, which accelerates the accumulation of lipids in arterial wall[12]. However, as a mechanical parameter, WSS could not directly reflect the process of atherosclerotic plaque growth. In comparison, in the calculation of LDL filtration rate, multiple mechanical (pressure, WSS) and physiological (LDL concentration, endothelial properties) parameters are included[13]. Resultantly, LDL filtration rate is not a linear function 
of WSS (Fig. 6). In carotid artery, LDL filtration rate has been proven to be more directly related with follow-up plaque growth than WSS[14]. Therefore, the application of LDL filtration rate in predicting plaque growth in intracranial arteries deserves further investigation.

The geometry of plaque and affected arterial segment has a strong effect on the risk of plaque rupture, as indicated by the distribution of high-WSS areas. In the patient-specific model, the third stenosis had a high average WSS value. Moreover, due to the coexistence of the bend, a high WSS belt extended distally from the throat of the third stenosis. High WSS $(>3 \mathrm{~Pa}$ ) has been proven to be related with the risks of plaque rupture[15]. Therefore, the risks of both plaque growth and rupture should be considered in severe stenoses (more than $85 \%$ in area). Moreover, the effect of arterial bend should be considered in estimating the risk of plaque rupture in severely stenosed intracranial arteries.

There are some limitations in this pilot study. Firstly, we were lack of the followup data. With follow-up imaging, the accuracies of WSS and LDL filtration rate in estimating the risk of atherosclerotic plaque growth could be compared in further studies. Secondly, we excluded the resistance of large and medium arteries in calculating flow resistances of distal branches. The exact value of intracranial arterial flow resistance depends on age, physiological and pathological conditions, therefore is difficult to derive[16]. Compared with 4D MRI measurement, CFD simulation correctly showed velocity distribution trends in branches of BA, but with higher velocity values, due to the inaccuracy in flow resistance[17]. The more accurate flow resistance value is important to improve the accuracy of CFD simulation. Thirdly, the LDL filtration model was based on some simplifications. In our calculation of the LDL filtration rate, endothelial properties were procured from literature. Pathologically, besides initial LDL filtration, atherosclerotic plaque formation development involves multiple physiological factors such as inflammation[18]. Therefore, in our results, the LDL filtration rate reflected the difference in the risk of plaque growth between different areas on arterial walls, but the values are not exactly patientspecific. Finally, in the tube models, stenoses were concentric. However, eccentric stenoses are common in the posterior cerebral circulation[19]. The effect of plaque eccentricity could be considered in further studies.

\section{Conclusion}

Tandem stenoses have hemodynamic effects on related clinical risks: 1. For tandem stenoses, their trans-stenotic pressure drops are independent and accumulative. Pressure drop is not obvious in less severe stenoses ( $<75 \%$ in area), but increases sharply with severity. The FF values of tandem stenoses are independent. 2. Compared with WSS, LDL filtration rate indicated more specifically the risky areas of atherosclerotic plaque growth. 3. In any severe stenosis ( $>85 \%$ in area), the risks of both plaque growth and rupture should be considered.

\section{Methods}

The study was based on four cylindrical tube models, and a patient-specific posterior cerebral circulation model. To estimate the risk of ischemia caused by tandem stenoses, we calculated the pressure drop and FF of each stenosis in four tube models 
and the patient-specific model. To estimate the risk of plaque growth, we calculated WSS and LDL filtration rate in the patient-specific model. To estimate the risk of plaque rupture, we observed the distribution of high WSS in the patient-specific model.

Tube models with tandem stenoses

Four cylindrical tube models were built with identical radius $(1.5 \mathrm{~mm})$ and length $(80 \mathrm{~mm})$. By setting the radius at stenosis throat as $0.75,0.6$ and $0.5 \mathrm{~mm}$ (diameter reductions, or diametric stenosis [20]: $50 \%, 60 \%$, and $66.7 \%$ ), we derived the stenoses with the severity (in area, defined as: $1-\frac{A_{S}}{A_{N}}$, where $A_{S}$ and $A_{N}$ denote the areas of cross sections at stenotic throat and in normal segment) of $75 \%, 84 \%$, and $89 \%$. The first model included only an $89 \%$ stenosis at $60 \mathrm{~mm}$ from the inlet. In other three models, the stenoses were added in sequence (Fig. 7a). In the second model, a $75 \%$ stenosis located at $20 \mathrm{~mm}$. In the third model, two $75 \%$ and $84 \%$ tandem stenoses located at $20 \mathrm{~mm}$ and $40 \mathrm{~mm}$. In the fourth model, the $74 \%, 84 \%$, and $89 \%$ tandem stenoses located at $20 \mathrm{~mm}, 40 \mathrm{~mm}$, and $60 \mathrm{~mm}$ respectively from the inlet. Meshing was performed on Ansys ICEM 17.0 (ANSYS Inc., USA). The maximum element length for meshing was $0.25 \mathrm{~mm}$ globally, and $0.1 \mathrm{~mm}$ at inlet and outlet.

Due to the shear-thinning effect, blood is essentially a non-Newtonian fluid. Newtonian fluid model was widely used but could be inaccurate[21]. In low-WSS areas, the difference in WSS between Newtonian and non-Newtonian models could exceed $10 \%[22]$. To eliminate the inaccuracy caused by Newtonian model, we adopted the Carreau-Yasuda non-Newtonian fluid model whose viscosity $\eta$ was a non-linear

function of shear strain rate $\gamma[23]: \eta(\gamma)=\eta_{\infty}+\left(\eta_{0}-\eta_{\infty}\right)\left(1+(\lambda \gamma)^{a}\right)^{(n-1) / a}$, in which $\eta_{0}=0.16 P a \cdot s, \eta_{\infty}=0.0035 P a \cdot s, \lambda=8.2 s, a=0.64$, and $n=0.2128$. CFD simulations were performed on the assumption of non-slip and solid arterial wall. In tube models, the flow rate and inlet pressure were $30 \mathrm{~cm} / \mathrm{s}$ and $120 \mathrm{mmHg}$ in diastolic simulation, whereas $60 \mathrm{~cm} / \mathrm{s}$ and $80 \mathrm{mmHg}$ in systolic simulation, according to Transcranial Doppler measurements in posterior cerebral circulation[24].

\section{Reconstruction of patient-specific model}

The imaging data was derived from the 3-dimensional rotational X-ray angiography (3DRA) images of a patient with ICAS, using an Integris BN 3000 biplane (Philips Medical System, Eindhoven, Netherlands). The resolution was $4 \mathrm{LP} / \mathrm{mm}$. The case was recruited to a prospective cohort study at Prince of Wales Hospital, Hong Kong, which was approved by local ethics committee, and the patient provided written informed consent. All data were anonymized. With high resolution, 3DRA is the gold standard of intracranial artery angiography. 3DRA imaging could preserve small distal branches to improve the accuracy of CFD simulation. In the original geometry (Fig. 7b, left), there were many unconnected arterial segments, unclosed faces and other topological errors. We extracted the segments connected with BA and discarded unconnected parts. The whole arterial structure included BA, bilateral posterior cerebral arteries (PCAs), and their distal small branches. For the outlets, only clear and continuous branches with diameters larger than $0.7 \mathrm{~mm}$ were preserved. Blurred or deformed distal segments of small arteries were deleted. The cross sections of outlets were cut perpendicularly to the local vessel directions. 
Finally, we smoothed the surfaces (Fig. 7b, right). For meshing, the minimum element size was $0.25 \mathrm{~mm}$ globally, $0.1 \mathrm{~mm}$ at inlet, and $0.05 \mathrm{~mm}$ at outlets. Totally there were 3290569 elements and 577814 nodes in the mesh.

\section{Patient-specific measurements and boundary conditions}

Derived from patient-specific measurement, blood pressure and flow resistance were used as inlet and outlet conditions in the CFD simulation of patient-specific arterial model.

The patient's blood pressure was measured continuously for 60 seconds. Five continuous cardiac cycles were extracted when the patient's blood pressure and heart rate remained stable. Cardiac cycles were segmented by the end-of-diastolic notches. The blood pressure was noted as $P(i, t(i))$, where $1 \leq i \leq 5$ is the sequence number of each cardiac cycle. In each cardiac cycle the time $t(i)$ satisfies: $0<t(i)<$ $T(i)$, where $T(i)$ is the time length of the $i$ th cardiac cycle. All cycle lengths were approximately $0.8 \mathrm{~s}$, with minor variations. We adjusted the time in each cycle: $t=t(i) \cdot \frac{0.8}{T(i)}$. Consequently, the new expression of pressure is: $P(i, t)$, where time lengths of the five cardiac cycles have been unified to $0.8 \mathrm{~s}$ : $0 \leq t \leq 0.8$. We then averaged the pressure curves in the five cardiac cycles (colored lines in Fig. 8): $P(t)=0.2 \sum_{i=1}^{5} P(i, t)$, and derived an averaged blood pressure curve ("Averaged Pressure" in Fig. 8). To provide a stable initial condition for CFD simulations, a $0.1 \mathrm{~s}$ translation was performed to avoid the sharp rise during initial systole. The derived pressure curve ("Translated Pressure" in Fig. 8) was repeated for three cardiac cycles (totally 2.4s) as the inlet condition on BA in CFD simulation.

It was difficult to measure the blood pressure or flow rate in distal small branches. Adopting flow resistance as outlet condition could avoid the errors caused by unreasonable parameter values, with flow rates of different outlets distributed automatically. Flow resistance was defined as: $R(i)=\frac{P(i)-P_{0}}{Q(i)}$, where $P(i)$ is the averaged pressure on $i$ th outlet, $P_{0}$ is the distal reference pressure, and $Q(i)$ is the outlet flow rate. Flow resistances were derived from patient-specific data. Firstly, time-averaged BA blood pressure $(77.9 \mathrm{mmHg})$ was derived from the integration of recorded blood pressure curve in 60s. Similarly, the time-averaged flow rate of BA was measured by carotid Doppler ultrasonography. The overall flow resistance was approximately calculated as the time-averaged pressure drop divided by the time-averaged flow rate. Since the flow resistance stems mainly from downstream arterioles, this value could be used as the estimation of overall distal flow resistance. Finally, the overall distal flow resistance was distributed to every outlet according to Murray's law which states that the flow distribution at an arterial bifurcation follows the principle of minimum work[25].

The CFD simulation lasted for three cardiac cycles (2.4s). The Carreau-Yasuda fluid model of blood was used. The non-slip and solid wall assumption was applied on arterial walls. The length of each time step was $0.005 \mathrm{~s}$. The convergence criterion was $10^{-4}$. Analysis was based on the results from the second cardiac cycle to eliminate any initial errors.

\section{Pressure drop and FF}

In the tube models, considering the unstable pressure distribution around the stenosis, the pressure drop of each stenosis was calculated as the difference between pres- 
sure values at $10 \mathrm{~mm}$ proximal and $20 \mathrm{~mm}$ distal to the stenotic throat. FF was calculated as the distal-to-proximal pressure ratio.

In the patient-specific model, three tandem stenoses existed in BA and PCA. The pressure was measured as the area-averaged value on 13 cross-sections in BA and PCA. There were 4 major arterial bends: located between the first and second cross-sections, as well at the 3rd, 8th, and 12th cross-sections (Fig. 2c). Especially, around the 12 th cross-section, a severe stenosis (89.8\% in area) coexisted with an arterial bend. For each stenosis, FF was calculated by dividing the distal pressure by the proximal pressure. The geometric (stenoses and bends) effects were estimated by observing the distribution of pressure on the cross-sections and the FF values of stenoses.

\section{WSS and LDL filtration rate in patient-specific model}

The tube models were idealistic and could not reflect the local variations of WSS and LDL filtration rate to disclose the risky areas of atherosclerotic plaque growth. Therefore, WSS and LDL filtration rate were calculated only in the patient-specific model.

In the stenotic segments, cylindrical projection was applied to extend the WSS distribution on the arterial walls to planes. We used the WSS scale from $0.5 \mathrm{~Pa}$ to $5 \mathrm{~Pa}$ referring to existing study[26]. The mean value and standard deviation of WSS were calculated.

Low-density lipoprotein (LDL) molecules filtrate from the lumen into the arterial wall through endothelial, which initiates the formation of atherosclerosis plaque and promotes its development. The filtration rate of LDL molecules in endothelial layer could reflect the risk of atherosclerotic development, and could be calculated with 3 -pore filtration model[6]. In the 3-pole model, there were two paths on the endothelial layer for LDL filtration: normal junctions and leaky junctions. Generated by abnormally low WSS, the leaky junctions accounted for over $90 \%$ of total LDL filtration into arterial walls. For simplification, only the LDL filtration in leaky junctions was considered. In the 3-pore model, the density of leaky junctions on arterial walls was calculated from the WSS value in CFD simulation, with blood LDL concentration from clinical recording, and material properties of LDL and endothelial from literature [6]. In the areas around the three stenoses, we estimated the risk of plaque growth by observing the distribution of areas with low WSS and high LDL filtration rate.

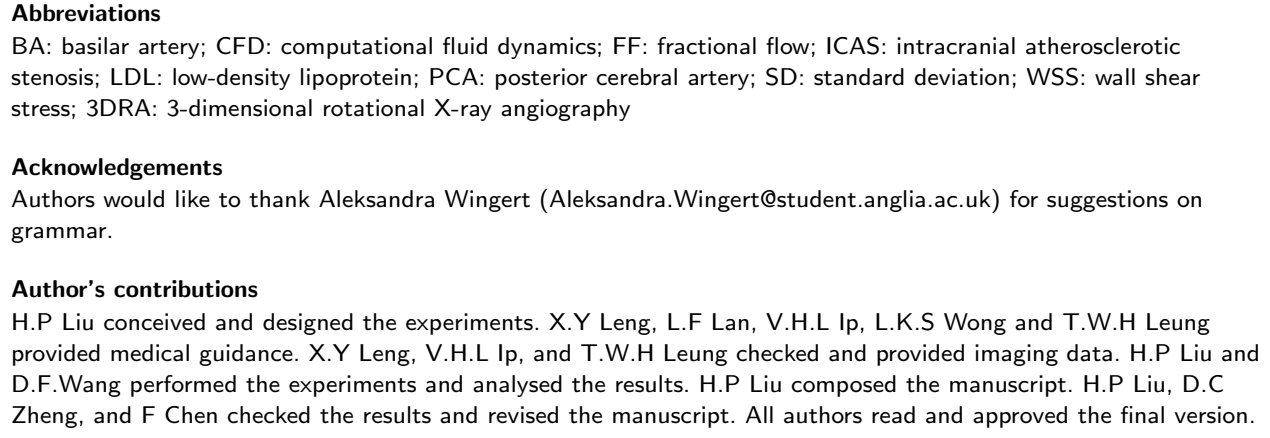


Funding

This work was supported by the Research Grant Council of Hong Kong (General Research Fund) [Grant Number 14117414]; by the Innovation and Technology Commission [Grant Number GHP/028/14SZ]; by Technology and Business Development Fund (TBF) [Grant Number TBF15MED004]; and by the National Natural Science Foundation of China [Grant Number:61828104].

Availability of data and materials

The data used or analyzed during the study are available from the corresponding author on reasonable request.

Ethics approval and consent to participate

Not applicable.

Consent for publication

Not applicable.

Competing interests

The authors declare that they have no competing interests.

\section{Author details}

${ }^{1}$ Research Center of Intelligent Healthcare, Faculty of Health and Life Science, Coventry University, CV1 5RW, Coventry, UK. ${ }^{2}$ Department of Imaging and Interventional Radiology, The Chinese University of Hong Kong, Prince of Wales Hospital, Hong Kong, China. ${ }^{3}$ Division of Neurology, Department of Medicine and Therapeutics, The Chinese University of Hong Kong, Prince of Wales Hospital, Hong kong, China. ${ }^{4}$ Research Institute for Frontier Science, Beihang University, 100191, Beijing, China. ${ }^{5}$ Beijing Advanced Innovation Center for Big Data-Based Precision Medicine, 100083, Beijing, China. ${ }^{6}$ Hangzhou Innovation Institute of Beihang University, 310000, Hangzhou, China. ${ }^{7}$ Department of Electrical and Electronic Engineering, Southern University of Science and Technology, 518055, Shenzhen, China.

\section{References}

1. Leung, T., Leung, H., Soo, Y.O., Mok, V.C., Wong, K.: The prognosis of acute symptomatic seizures after ischaemic stroke. Journal of Neurology, Neurosurgery \& Psychiatry 88(1), 86-94 (2017)

2. Mpotsaris, A., Bussmeyer, M., Buchner, H., Weber, W.: Clinical outcome of neurointerventional emergency treatment of extra-or intracranial tandem occlusions in acute major stroke: antegrade approach with wallstent and solitaire stent retriever. Clinical neuroradiology 23(3), 207-215 (2013)

3. Banerjee, C., Chimowitz, M.I.: Stroke caused by atherosclerosis of the major intracranial arteries. Circulation research 120(3), 502-513 (2017)

4. Liebeskind, D.S., Feldmann, E.: Fractional flow in cerebrovascular disorders. Interventional neurology 1(2), 87-99 (2012)

5. Peiffer, V., Sherwin, S.J., Weinberg, P.D.: Does low and oscillatory wall shear stress correlate spatially with early atherosclerosis? a systematic review. Cardiovascular research 99(2), 242-250 (2013)

6. Olgac, U., Knight, J., Poulikakos, D., Saur, S.C., Alkadhi, H., Desbiolles, L.M., Cattin, P.C., Kurtcuoglu, V.: Computed high concentrations of low-density lipoprotein correlate with plaque locations in human coronary arteries. Journal of biomechanics 44(13), 2466-2471 (2011)

7. Miao, Z., Liebeskind, D.S., Lo, W., Liu, L., Pu, Y., Leng, X., Song, L., Xu, X., Jia, B., Gao, F., et al.: Fractional flow assessment for the evaluation of intracranial atherosclerosis: a feasibility study. Interventional neurology 5(1-2), 65-75 (2016)

8. Yin, X., Huang, X., Feng, Y., Tan, W., Liu, H., Huo, Y.: Interplay of proximal flow confluence and distal flow divergence in patient-specific vertebrobasilar system. PloS one 11(7) (2016)

9. Liu, J., Yan, Z., Pu, Y., Shiu, W.-S., Wu, J., Chen, R., Leng, X., Qin, H., Liu, X., Jia, B., et al.: Functional assessment of cerebral artery stenosis: a pilot study based on computational fluid dynamics. Journal of Cerebral Blood Flow \& Metabolism 37(7), 2567-2576 (2017)

10. Guppy, K.H., Charbel, F.T., Loth, F., Ausman, J.I.: Hemodynamics of in-tandem stenosis of the internal carotid artery: when is carotid endarterectomy indicated? Surgical neurology 54(2), 145-154 (2000)

11. Kolli, K.K., Banerjee, R.K., Peelukhana, S.V., Helmy, T.A., Leesar, M.A., Arif, I., Schneeberger, E.W., Hand, D., Succop, P., Gottliebson, W.M., et al.: Influence of heart rate on fractional flow reserve, pressure drop coefficient, and lesion flow coefficient for epicardial coronary stenosis in a porcine model. American Journal of Physiology-Heart and Circulatory Physiology 300(1), 382-387 (2011)

12. Schirmer, C.M., Malek, A.M.: Estimation of wall shear stress dynamic fluctuations in intracranial atherosclerotic lesions using computational fluid dynamics. Neurosurgery 63(2), 326-335 (2008)

13. Dong, J., Wong, K.K., Tu, J.: Effect of local wall shear stress on low-density lipoprotein transportation in atherosclerotic carotid bifurcation. In: 2012 2nd International Conference on Biomedical Engineering and Technology, pp. 62-67 (2012)

14. Liu, H., Gong, Y., Leng, X., Xia, L., Wong, K.S., Ou, S., Leung, T.W., Wang, D., Shi, L.: Estimating current and long-term risks of coronary artery in silico by fractional flow reserve, wall shear stress and low-density lipoprotein filtration rate. Biomedical Physics \& Engineering Express 4(2), 025006 (2018)

15. Dolan, J.M., Kolega, J., Meng, H.: High wall shear stress and spatial gradients in vascular pathology: a review. Annals of biomedical engineering 41(7), 1411-1427 (2013)

16. Hauck, E.F., Apostel, S., Hoffmann, J.F., Heimann, A., Kempski, O.: Capillary flow and diameter changes during reperfusion after global cerebral ischemia studied by intravital video microscopy. Journal of Cerebral Blood Flow \& Metabolism 24(4), 383-391 (2004)

17. Cebral, J.R., Putman, C.M., Alley, M.T., Hope, T., Bammer, R., Calamante, F.: Hemodynamics in normal cerebral arteries: qualitative comparison of $4 \mathrm{~d}$ phase-contrast magnetic resonance and image-based computational fluid dynamics. Journal of engineering mathematics 64(4), 367 (2009) 
18. Rahman, M.S., Murphy, A.J., Woollard, K.J.: Effects of dyslipidaemia on monocyte production and function in cardiovascular disease. Nature Reviews Cardiology 14(7), 387 (2017)

19. Zhu, X., Liu, L., He, X., Zhang, X., Hu, L., Du, B., Wang, W., Jiang, W., Liu, Z.: Wall thickening pattern in atherosclerotic basilar artery stenosis. Neurological Sciences 37(2), 269-276 (2016)

20. Ferguson, G.G., Eliasziw, M., Barr, H.W., Clagett, G.P., Barnes, R.W., Wallace, M.C., Taylor, D.W., Haynes, R.B., Finan, J.W., Hachinski, V.C., et al.: The north american symptomatic carotid endarterectomy trial: surgical results in 1415 patients. Stroke 30(9), 1751-1758 (1999)

21. AlArgha, H.M., Hamdan, M.O., Elshawarby, A., Aziz, W.H.: Cfd sensitivity study for newtonian viscosity model in cerebral aneurysms. In: Eleventh International Conference on Computational Fluid Dynamics in the Minerals and Process Industries, Melbourne, Australia (2015)

22. Perktold, K., Hofer, M., Rappitsch, G., Loew, M., Kuban, B., Friedman, M.: Validated computation of physiologic flow in a realistic coronary artery branch. Journal of biomechanics 31(3), $217-228$ (1997)

23. Bernabeu, M.O., Nash, R.W., Groen, D., Carver, H.B., Hetherington, J., Krüger, T., Coveney, P.V.: Impact of blood rheology on wall shear stress in a model of the middle cerebral artery. Interface Focus 3(2), 20120094 (2013)

24. Forteza, A., Krejza, J., Koch, S., Babikian, V.: Ultrasound imaging of cerebrovascular disease. Imaging Cerebrovascular Disease. Philadelphia: Butterworth-Heinemann, 3-35 (2003)

25. Doddasomayajula, R., Chung, B., Hamzei-Sichani, F., Putman, C., Cebral, J.: Differences in hemodynamics and rupture rate of aneurysms at the bifurcation of the basilar and internal carotid arteries. American Journal of Neuroradiology 38(3), 570-576 (2017)

26. Samady, H., Eshtehardi, P., McDaniel, M.C., Suo, J., Dhawan, S.S., Maynard, C., Timmins, L.H., Quyyumi, A.A., Giddens, D.P.: Coronary artery wall shear stress is associated with progression and transformation of atherosclerotic plaque and arterial remodeling in patients with coronary artery disease. Circulation 124(7), 779-788 (2011)

Figures

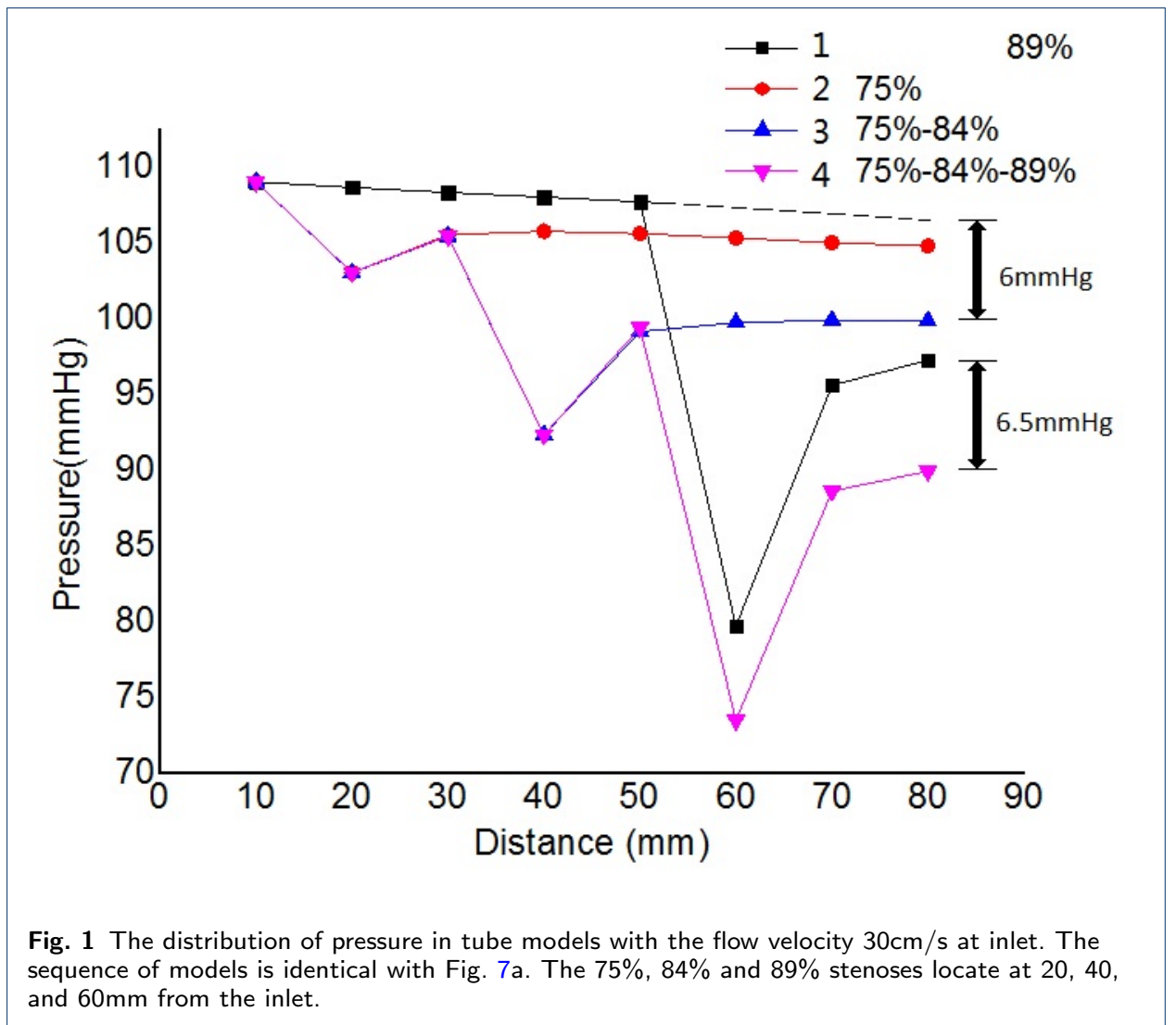




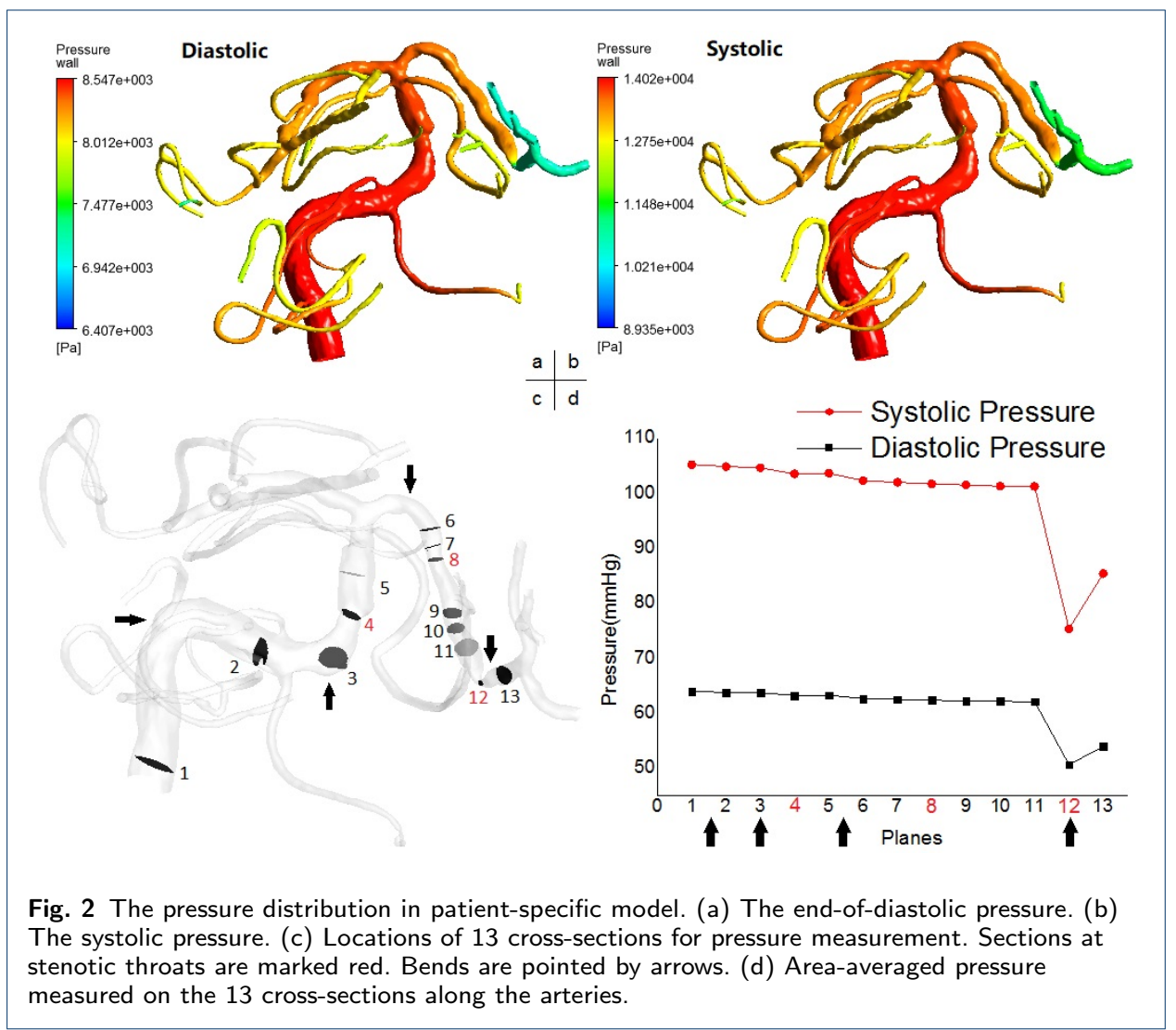

Table 1 Diastolic/systolic FF values of the stenoses in the tube models.

\begin{tabular}{lccc}
\hline \multirow{2}{*}{ Models } & \multicolumn{3}{c}{ Diastolic/systolic FF values of each stenosis } \\
\cline { 2 - 4 } & $\mathbf{7 5 \%}$ & $\mathbf{8 4 \%}$ & $\mathbf{8 9 \%}$ \\
\hline $\mathbf{1}$ & & & $0.844 / 0.717$ \\
$\mathbf{2}$ & $0.956 / 0.917$ & & \\
$\mathbf{3}$ & $0.955 / 0.916$ & $0.916 / 0.834$ & \\
$\mathbf{4}$ & $0.955 / 0.916$ & $0.920 / 0.839$ & $0.844 / 0.653$ \\
\hline
\end{tabular}

Table 2 Severity in area, pressure distribution, and FF value of the three stenoses in the patient-specific model.

\begin{tabular}{llccc}
\hline Stenosis & $\mathbf{1}$ & $\mathbf{2}$ & $\mathbf{3}$ \\
\hline Cross-section area in normal segment $\left.\mathbf{( m m}^{2}\right)$ & 9.59 & 4.81 & 3.64 \\
Cross-section area at stenotic throat $\left(\mathbf{m m}^{2}\right)$ & 4.20 & 2.34 & 3.73 \\
Severity (in area) & & $56.2 \%$ & $51.3 \%$ & $89.8 \%$ \\
\hline \multirow{2}{*}{ Diastolic pressure $(\mathbf{m m H g})$} & Proximal & 63.70 & 62.64 & 62.10 \\
& Stenotic throat & 63.24 & 62.36 & 50.67 \\
& Distal & 63.28 & 62.24 & 53.93 \\
Diastolic FF & & 0.99 & 0.99 & 0.87 \\
\hline \multirow{3}{*}{ Systolic pressure $(\mathbf{m m H g})$} & Proximal & 104.58 & 102.26 & 101.19 \\
& Stenotic throat & 103.48 & 101.65 & 75.33 \\
Systolic FF & Distal & 103.60 & 101.45 & 85.36 \\
& & 0.99 & 0.99 & 0.84 \\
\hline
\end{tabular}




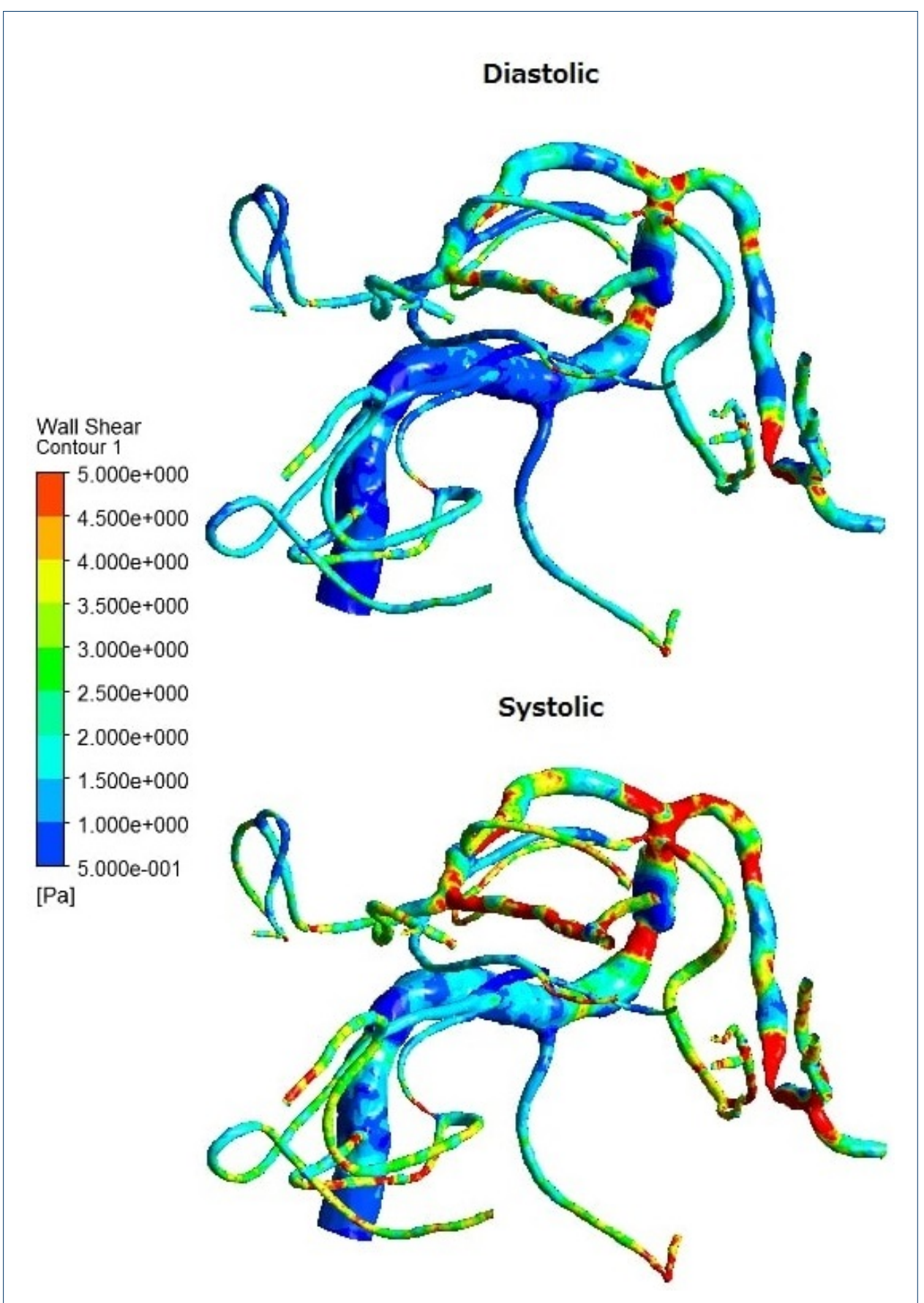

Fig. 3 The diastolic and systolic distribution of WSS in the patient-specific model.

Table 3 Diastolic and systolic WSS distribution (mean \pm standard deviation) on the whole arterial wall and in three stenotic areas (shown in Fig. 4) of the patient-specific model.

\begin{tabular}{lcc}
\hline Location & Diastolic WSS $\mathbf{( P a )}$ & Systolic WSS $\mathbf{( P a )}$ \\
\hline Wall & $1.45 \pm 1.65$ & $2.55 \pm 2.79$ \\
Stenosis 1 & $1.56 \pm 1.44$ & $2.94 \pm 2.60$ \\
Stenosis 2 & $1.77 \pm 1.03$ & $3.14 \pm 1.87$ \\
Stenosis 3 & $7.28 \pm 8.90$ & $12.89 \pm 14.46$ \\
\hline
\end{tabular}




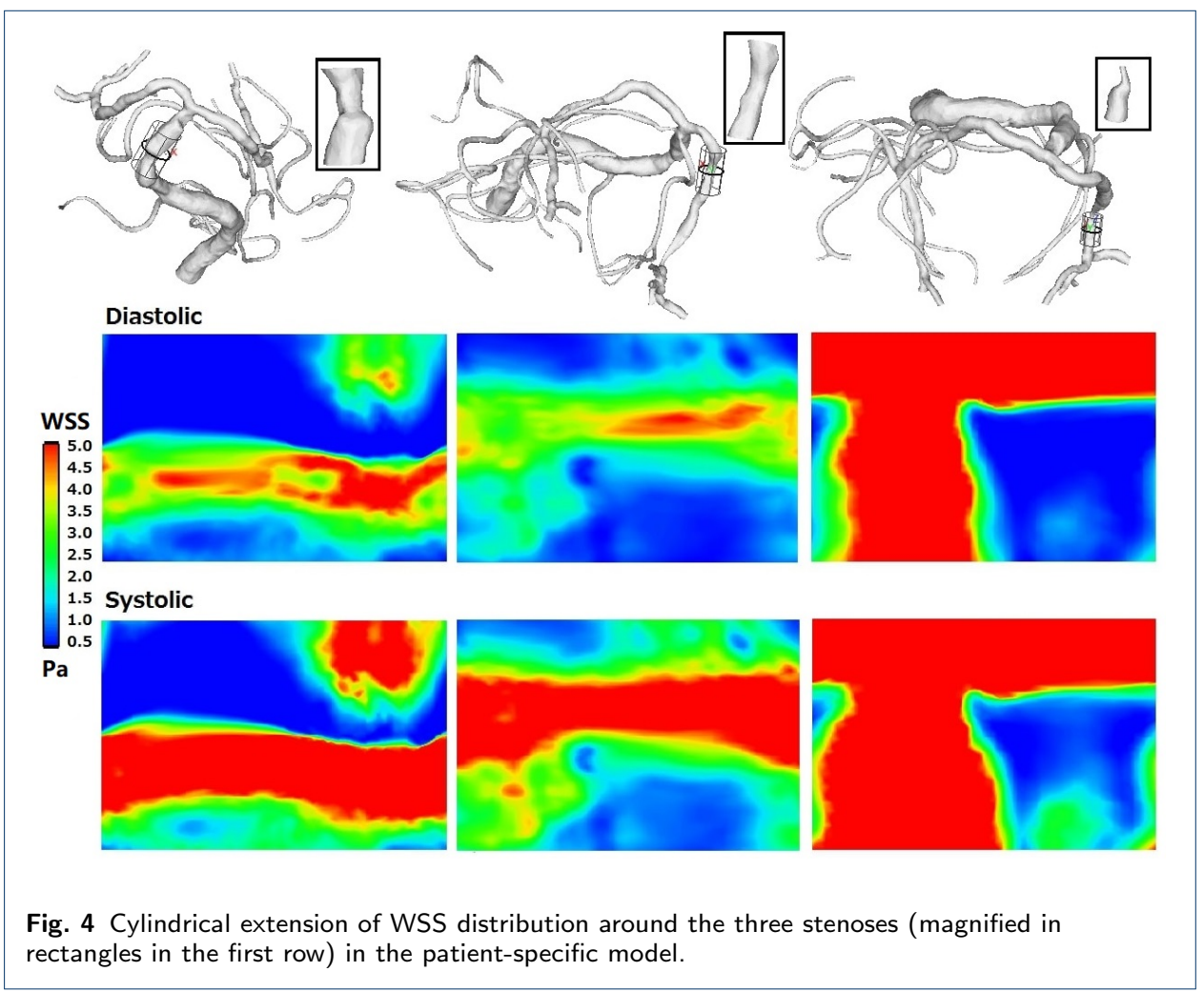




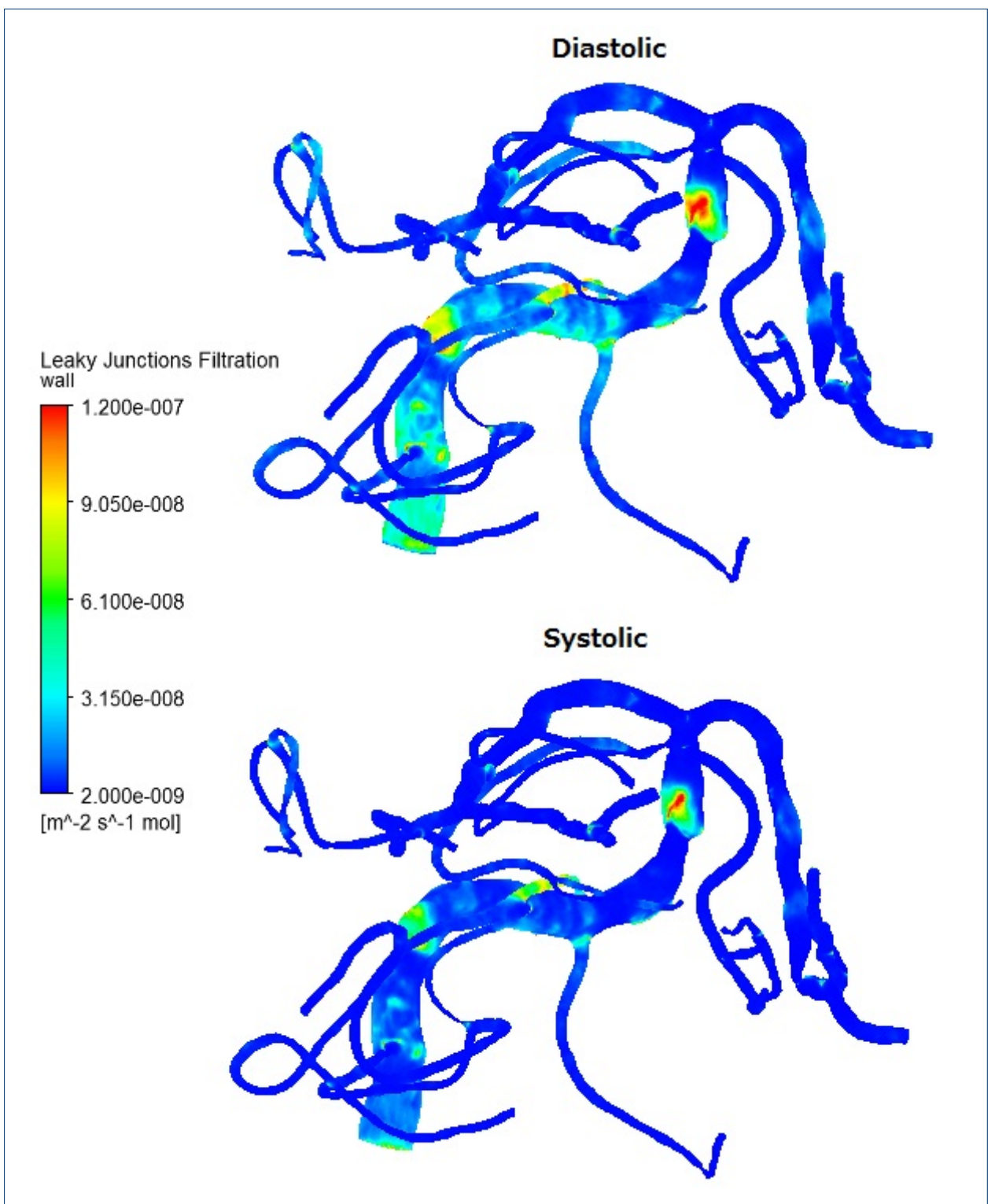

Fig. 5 The diastolic and systolic distribution of LDL filtration rate. 


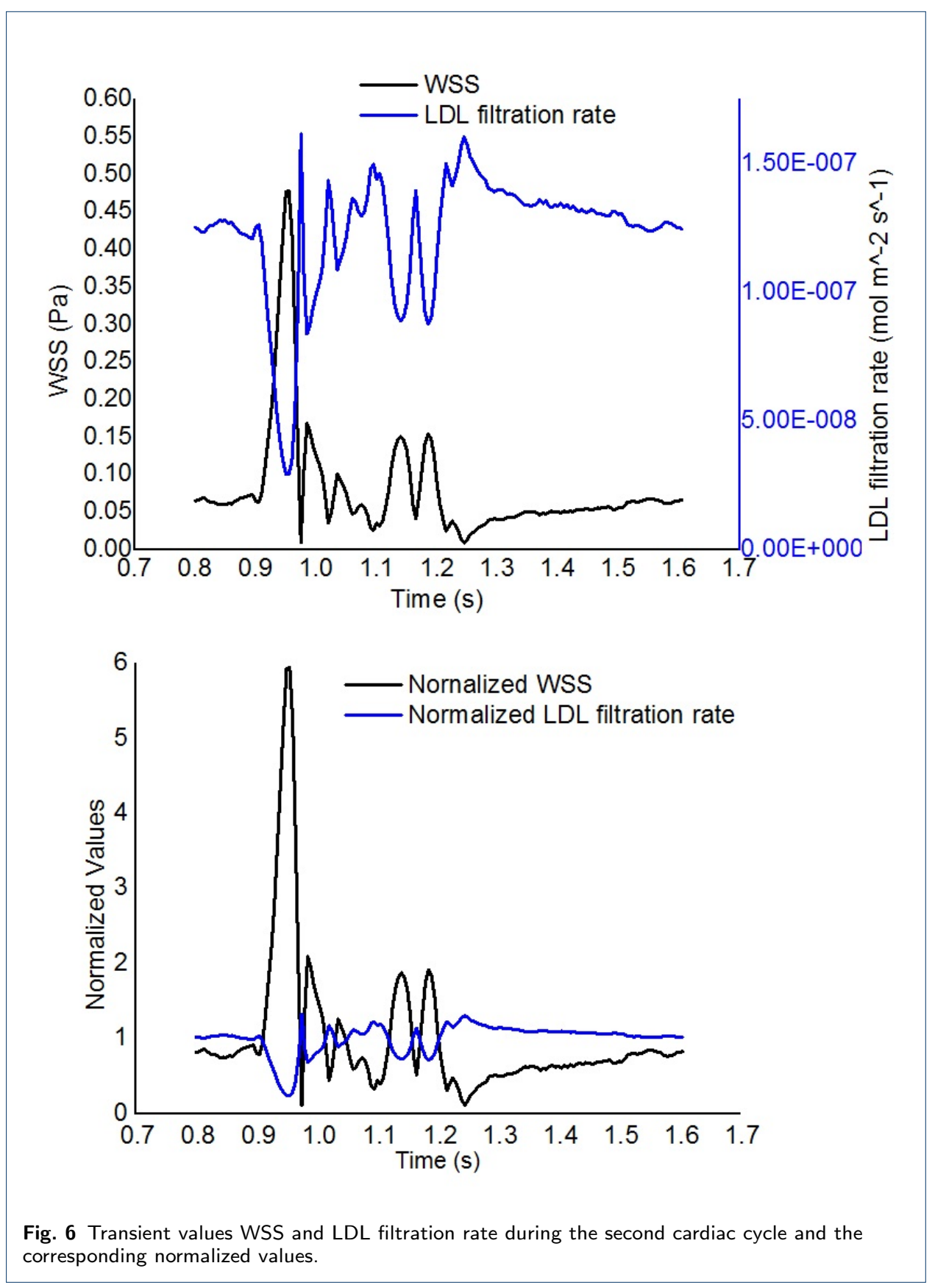




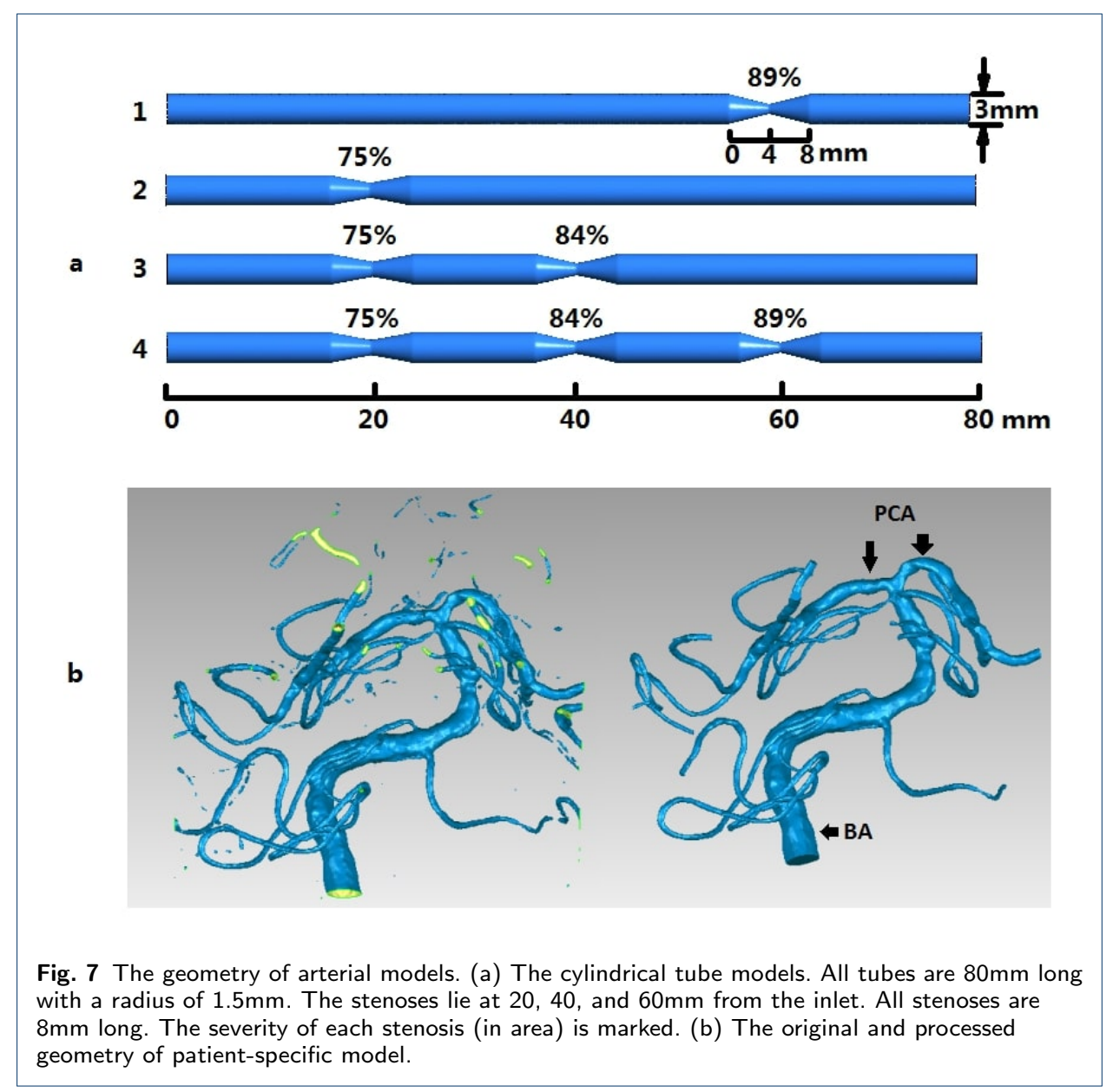




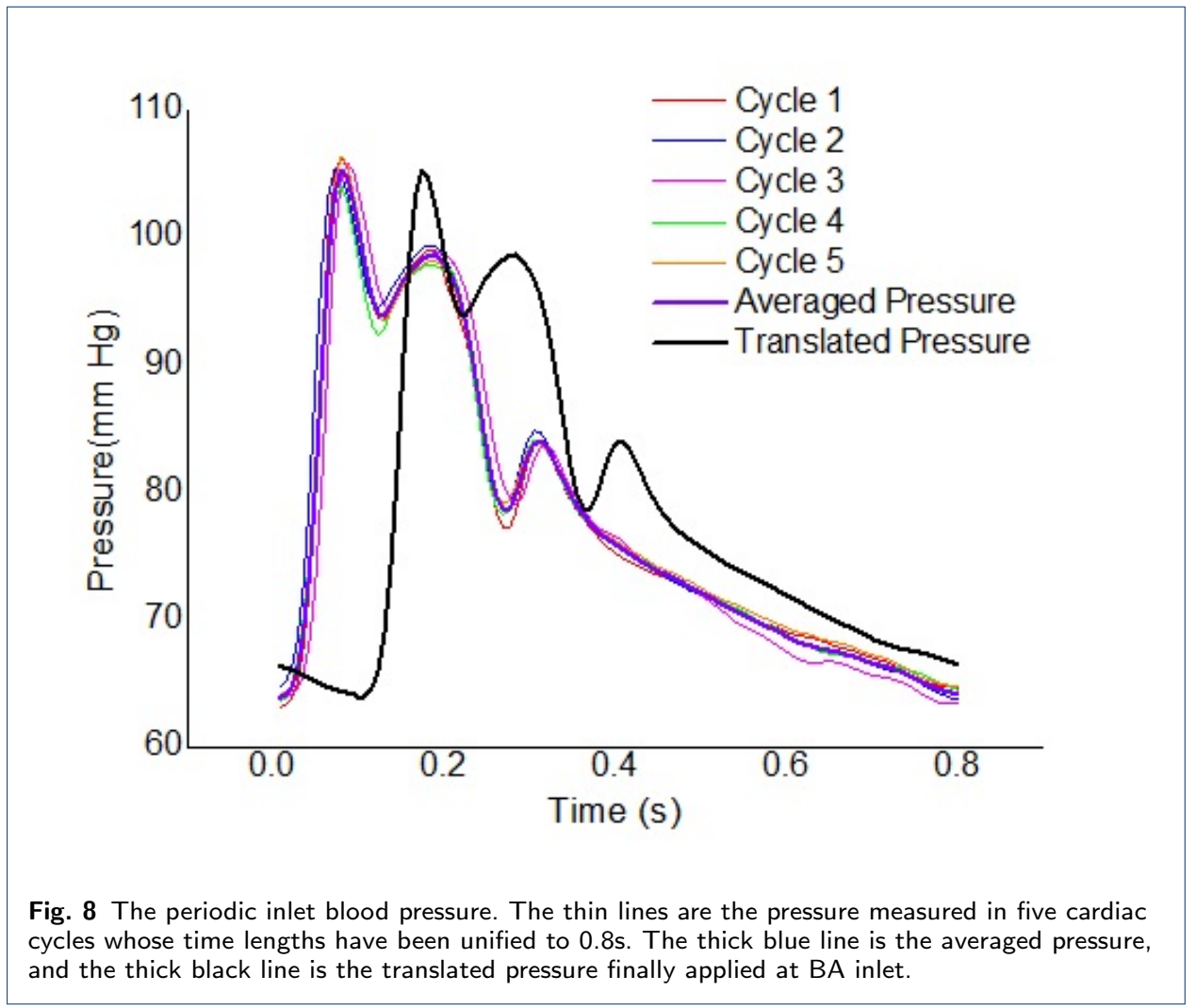


Figures

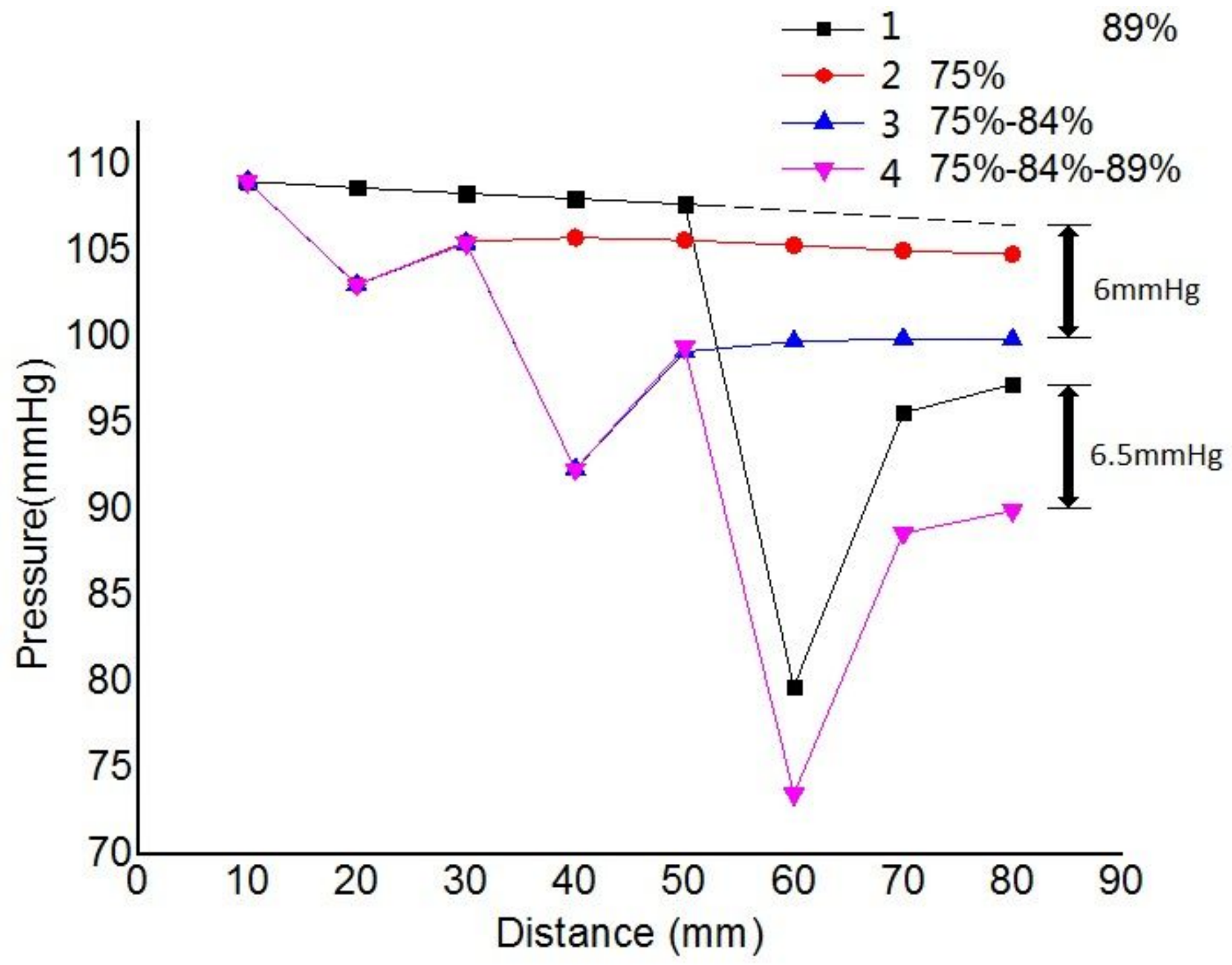

Figure 1

The distribution of pressure in tube models with the flow velocity $30 \mathrm{~cm} / \mathrm{s}$ at inlet. The sequence of models is identical with Fig. 7 a. The $75 \%, 84 \%$ and $89 \%$ stenoses locate at 20,40 , and $60 \mathrm{~mm}$ from the inlet 


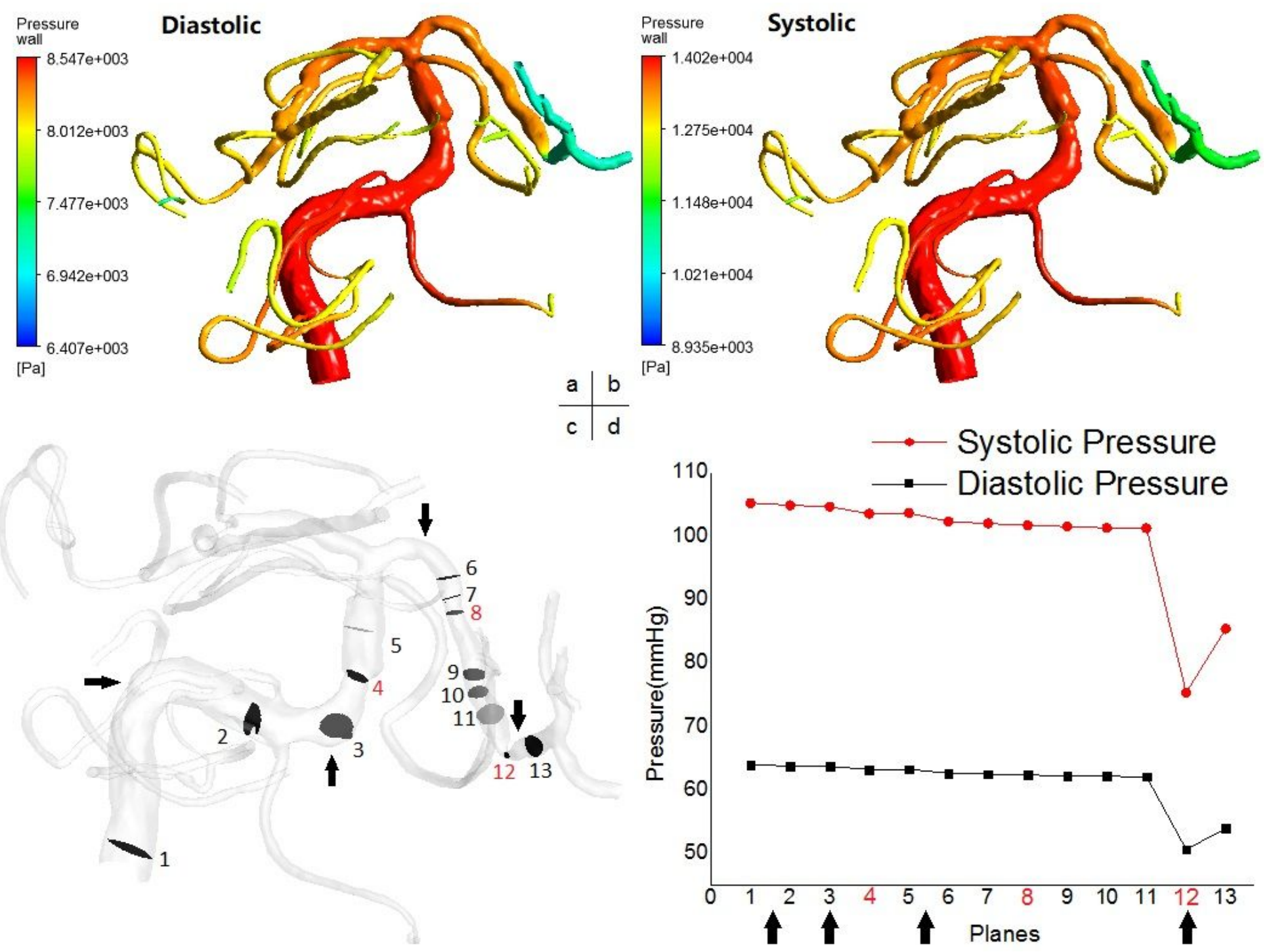

Figure 2

The pressure distribution in patient-specific model. (a) The end-of-diastolic pressure. (b) The systolic pressure. (c) Locations of 13 cross-sections for pressure measurement. Sections at stenotic throats are marked red. Bends are pointed by arrows. (d) Area-averaged pressure measured on the 13 cross-sections along the arteries. 


\section{Diastolic}

Wall Shear

Contour 1
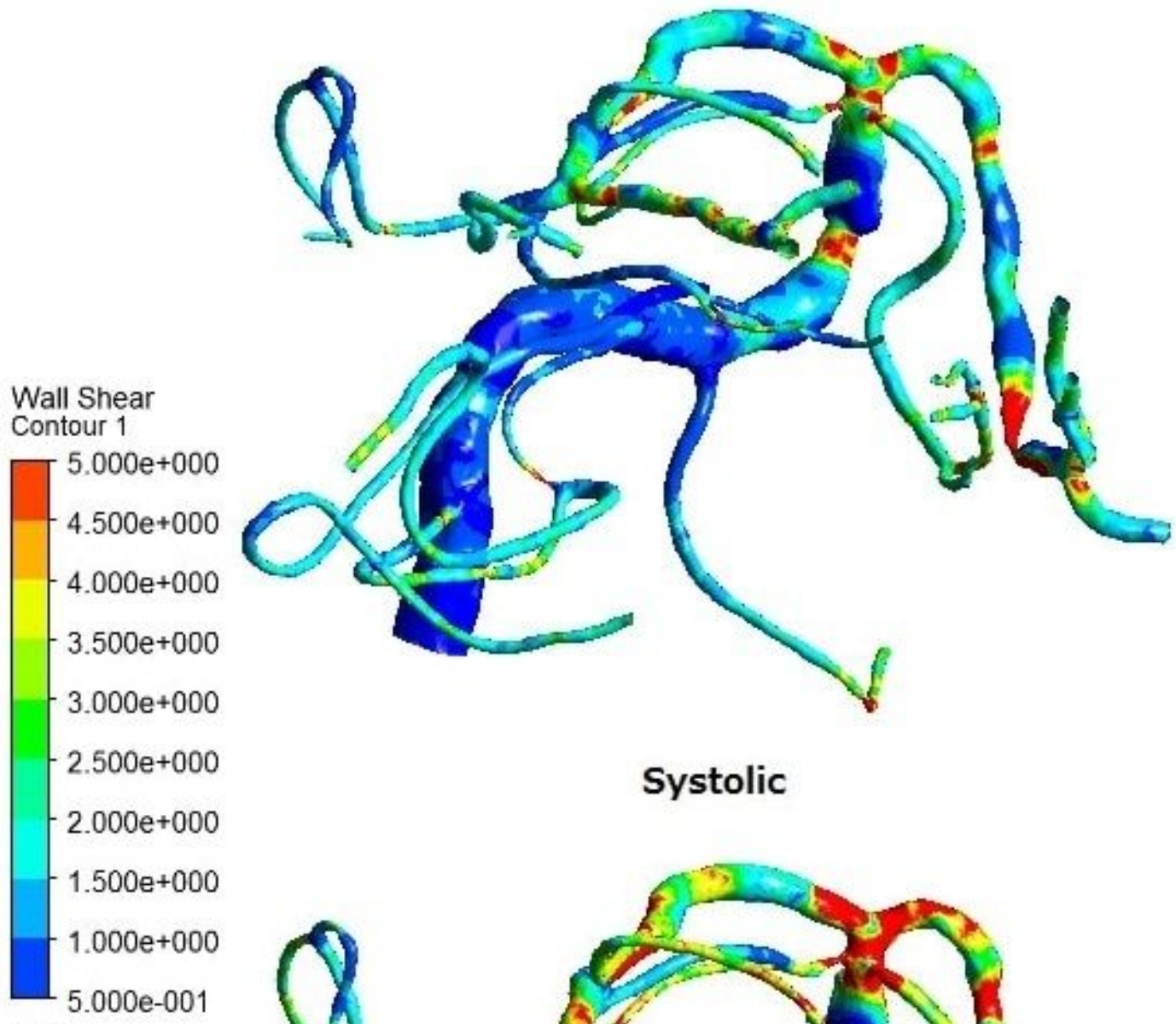

Systolic

[Pa]

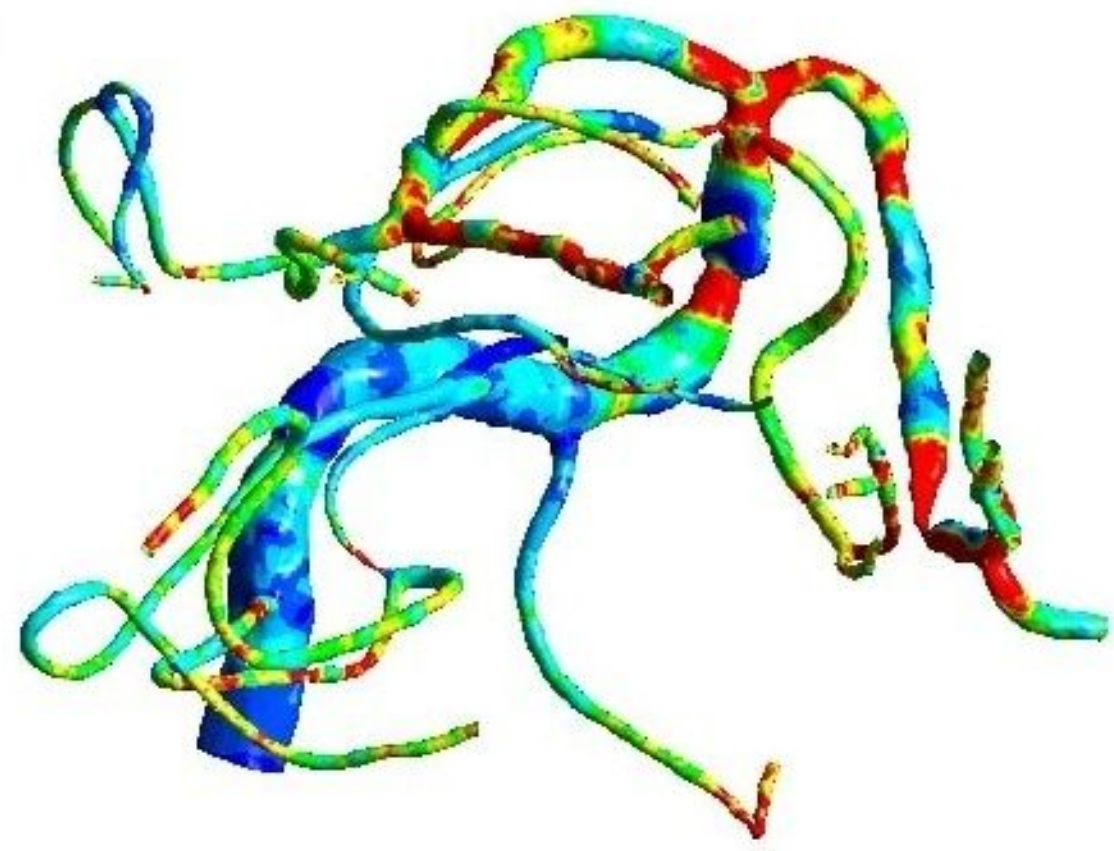

Figure 3

The diastolic and systolic distribution of WSS in the patient-specific model. 


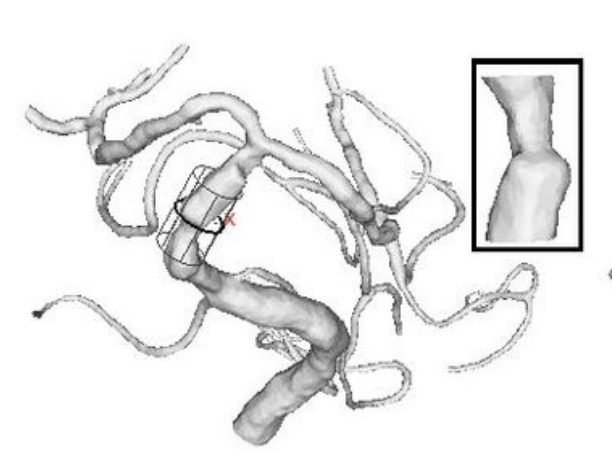

\section{Diastolic}

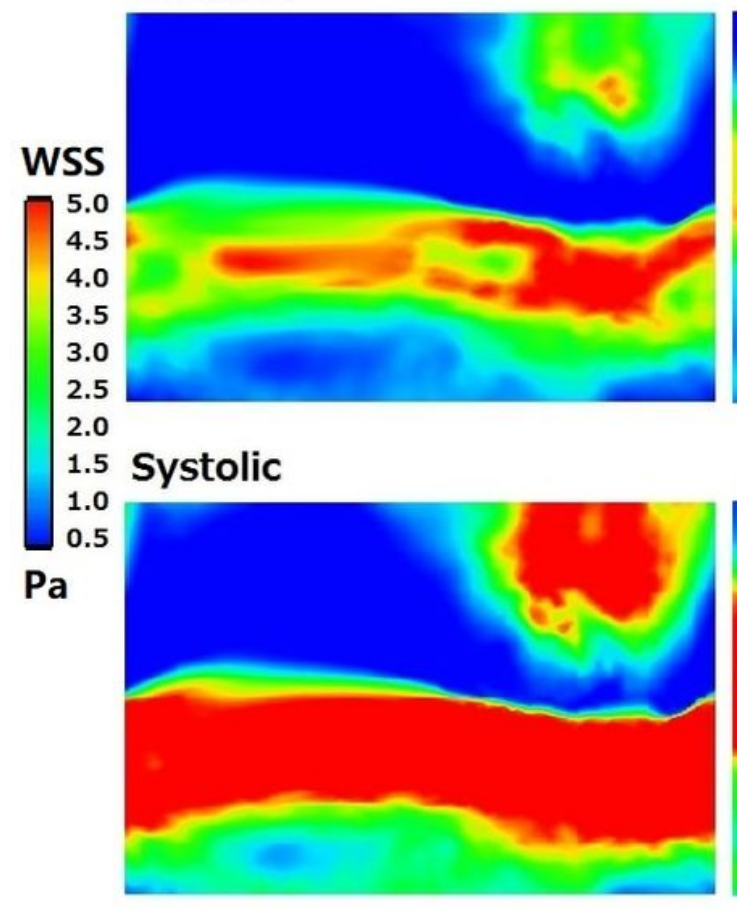

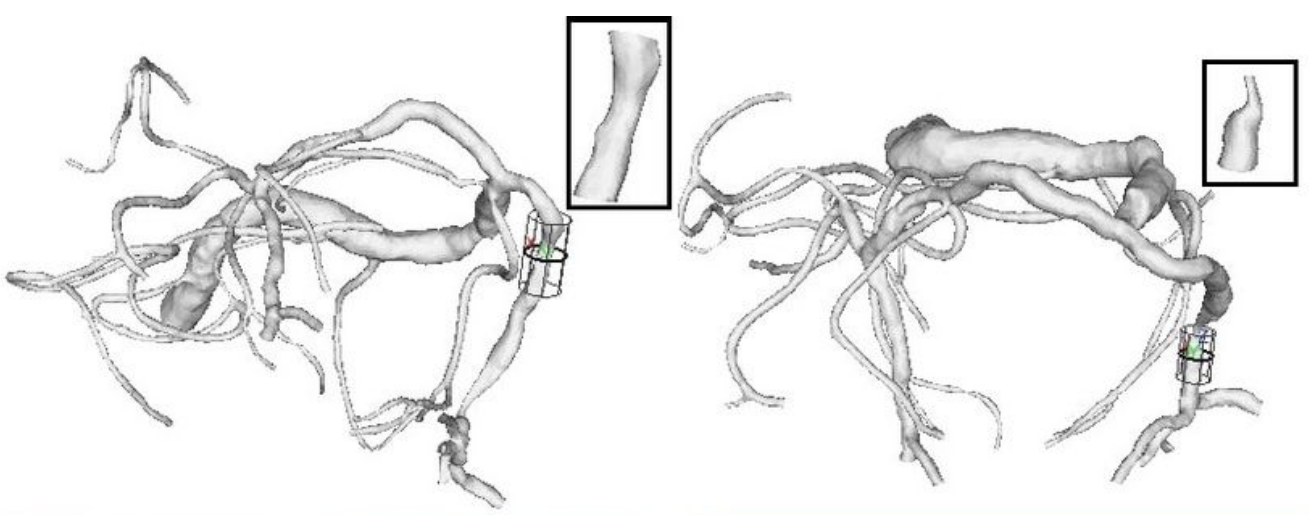
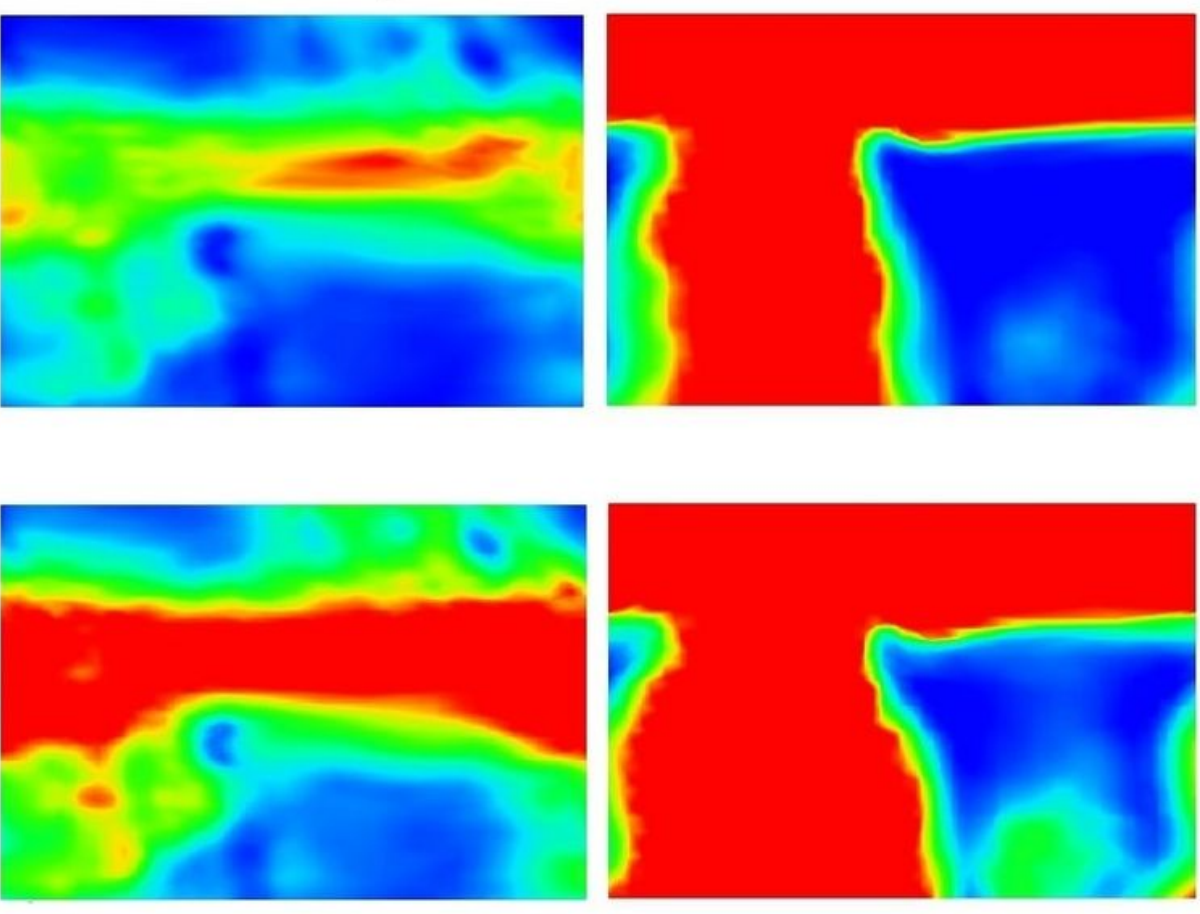

Figure 4

Cylindrical extension of WSS distribution around the three stenoses (magnified in rectangles in the first row) in the patient-specific model. 


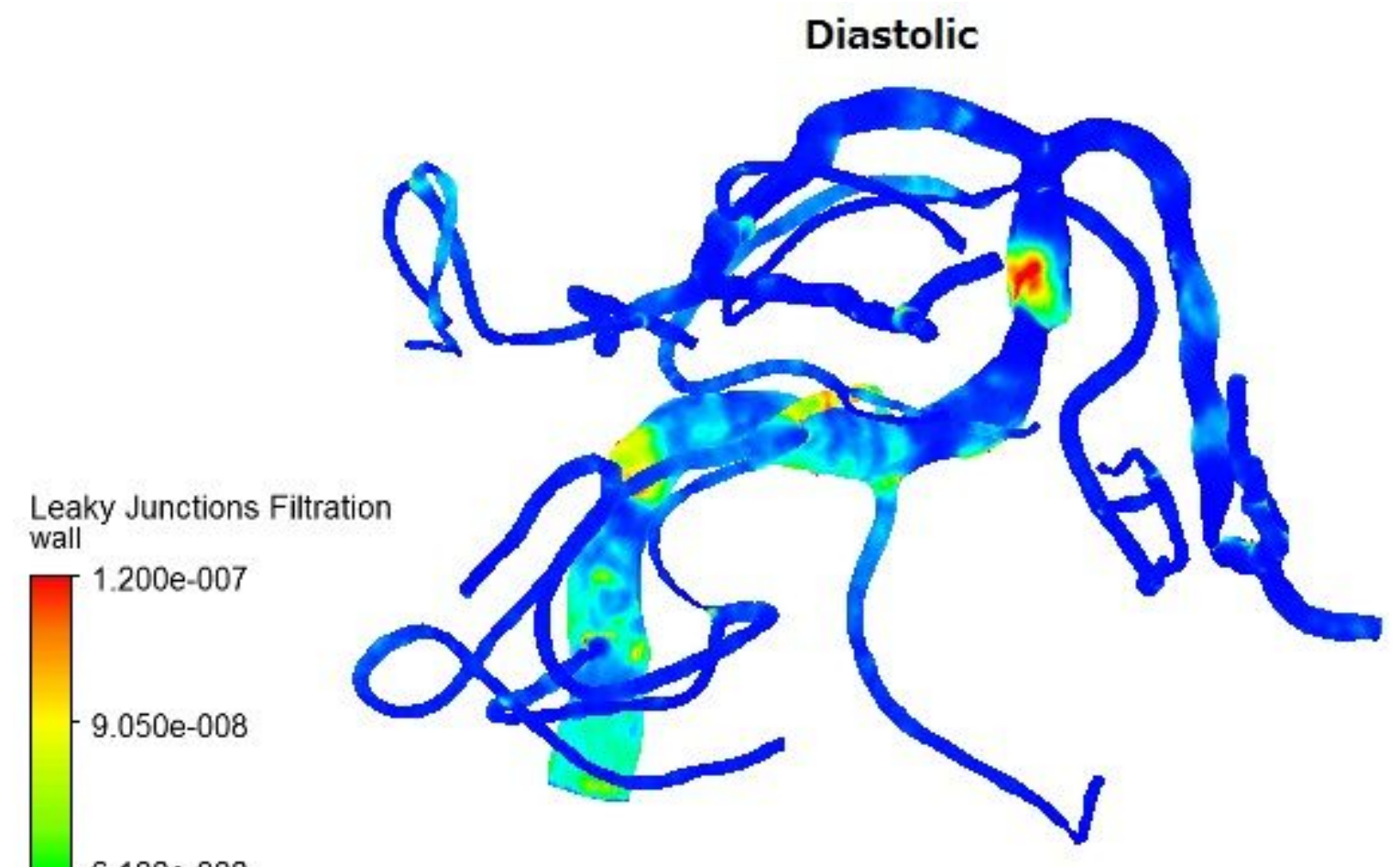

$6.100 \mathrm{e}-008$

\section{Systolic}

$-3.150 \mathrm{e}-008$

2.000e-009 [m^-2 $\left.\mathrm{s}^{\wedge}-1 \mathrm{~mol}\right]$

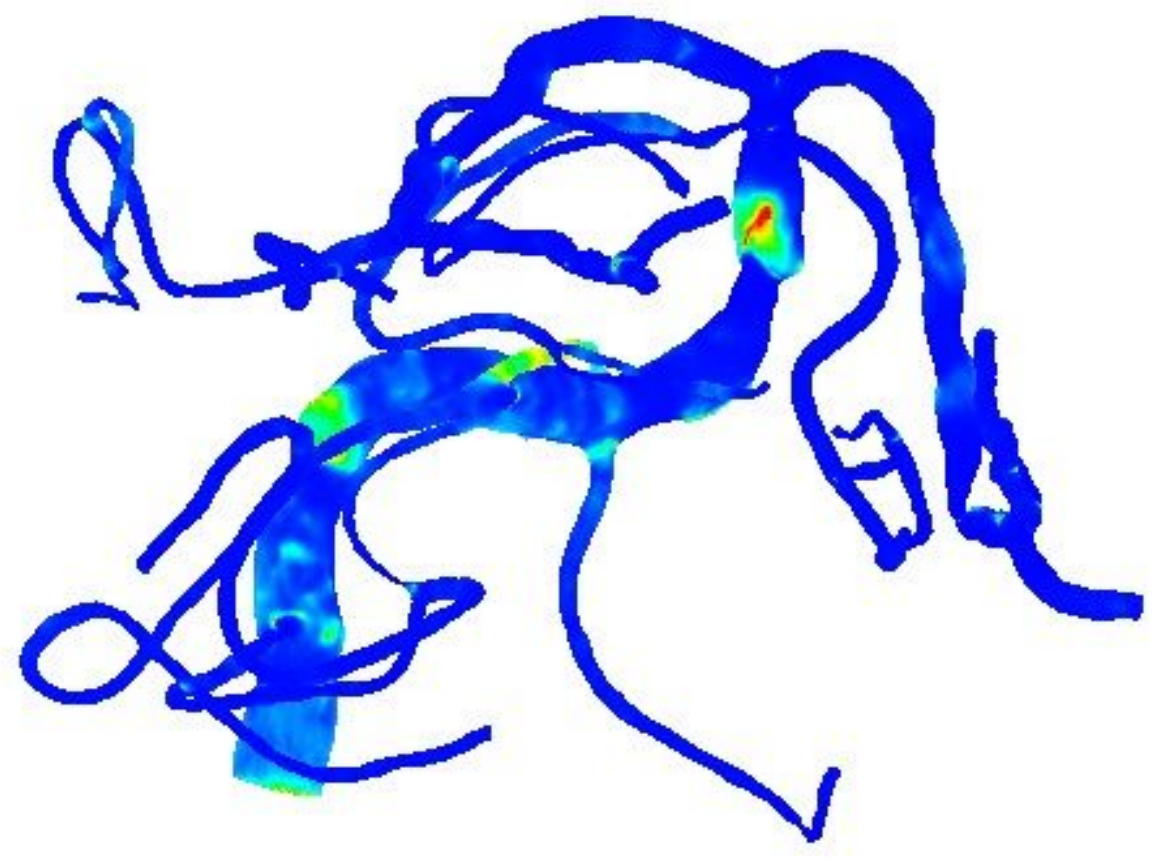

Figure 5

The diastolic and systolic distribution of LDL filtration rate. 

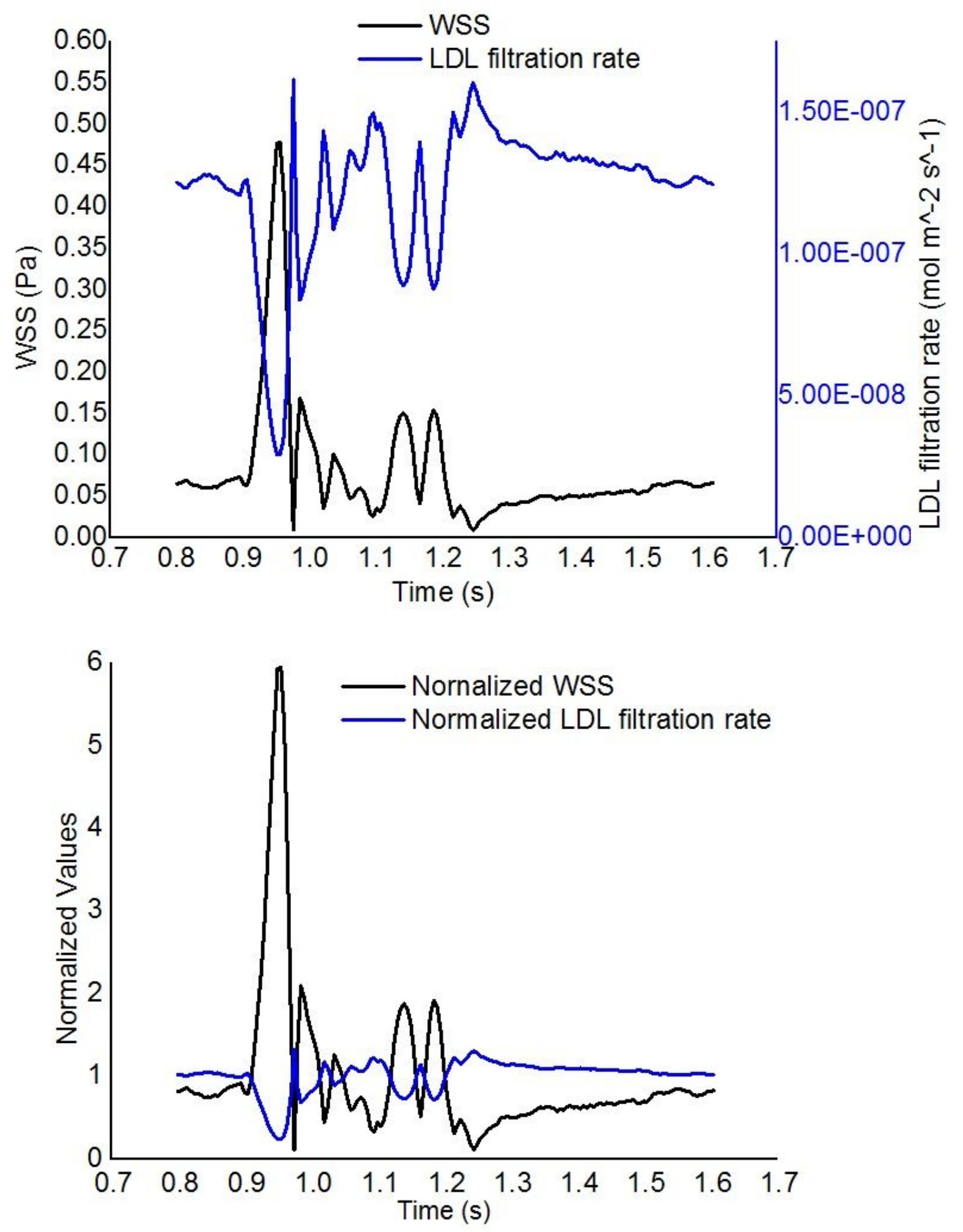

Figure 6

Transient values WSS and LDL filtration rate during the second cardiac cycle and the corresponding normalized values. 

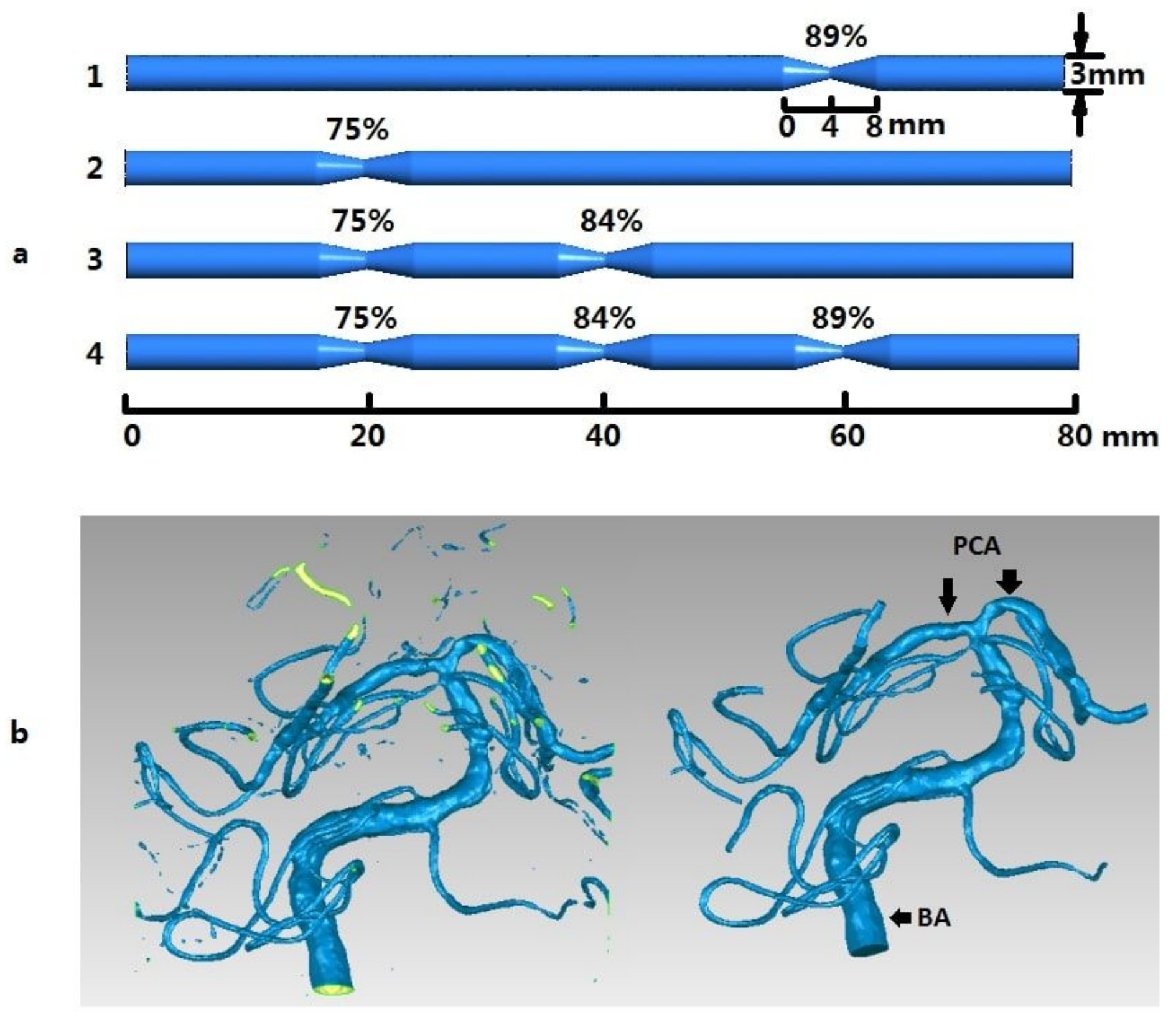

\section{Figure 7}

The geometry of arterial models. (a) The cylindrical tube models. All tubes are $80 \mathrm{~mm}$ long with a radius of $1.5 \mathrm{~mm}$. The stenoses lie at 20,40 , and $60 \mathrm{~mm}$ from the inlet. All stenoses are $8 \mathrm{~mm}$ long. The severity of each stenosis (in area) is marked. (b) The original and processed geometry of patient-specific model. 


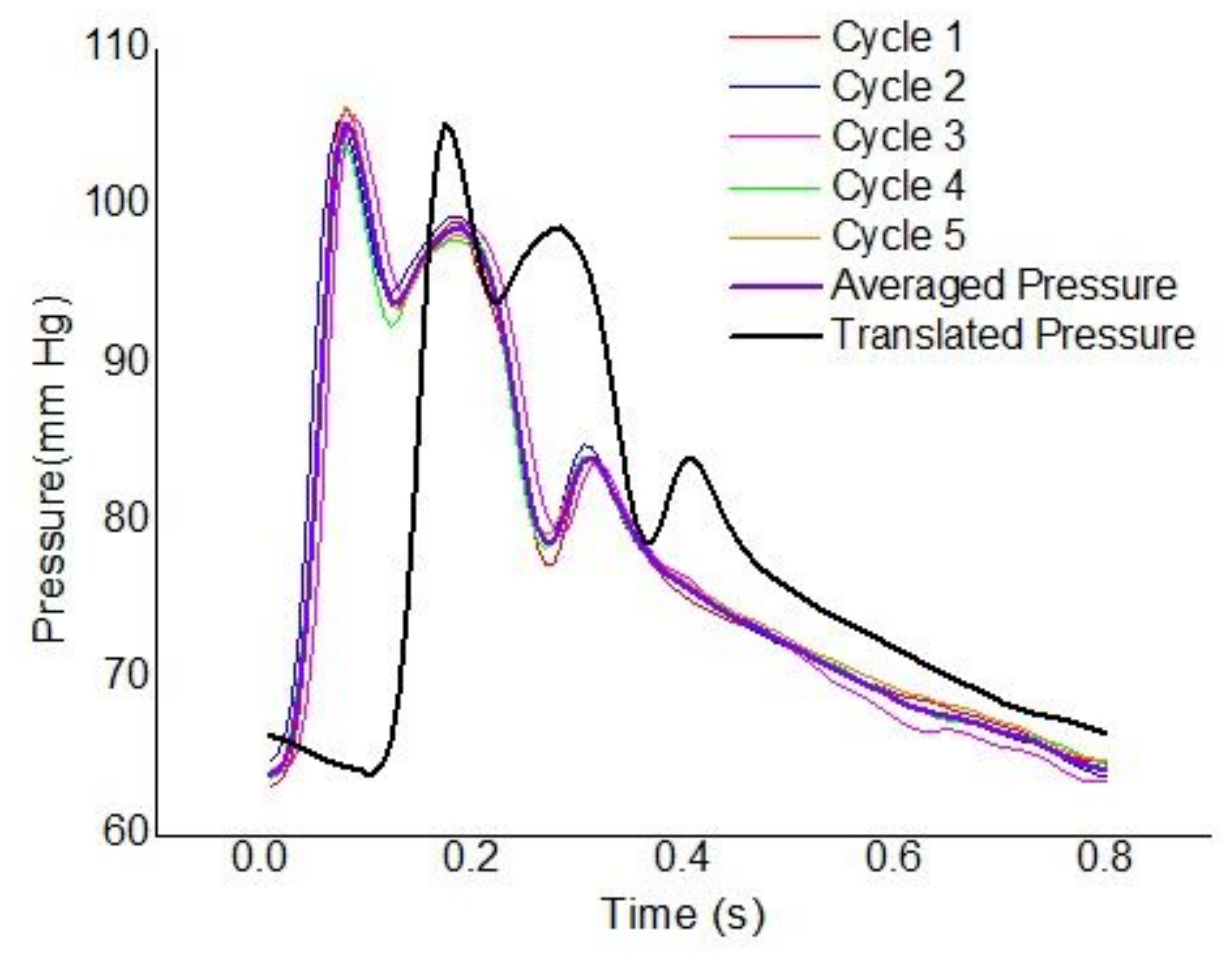

Figure 8

The periodic inlet blood pressure. The thin lines are the pressure measured in five cardiac cycles whose time lengths have been unified to $0.8 \mathrm{~s}$. The thick blue line is the averaged pressure, and the thick black line is the translated pressure finally applied at BA inlet. 